\title{
Limnological features and models of chlorophyll- $a$ in 30 lakes located in the lower Mackenzie River basin, Northwest Territories (Canada)
}

\author{
Fortune E. OGBEBO, Marlene S. EVANS*, Robert B. BRUA ${ }^{1)}$ and Jonathan J. KEATING \\ Aquatic Ecosystem Protection Research Division (AEPRD), Water Science and Technology Directorate, Environment Canada, 11 \\ Innovation Blvd., Saskatoon, SK, Canada S7N 3H5 \\ ${ }^{1)}$ Aquatic Ecosystem Impacts Research Division (AEIRD), Water Science and Technology Directorate, Environment Canada, 11 \\ Innovation Blvd., Saskatoon, SK, Canada S7N 3H5 \\ *e-mail corresponding author: marlene.evans@ec.gc.ca
}

\begin{abstract}
Limnological variables from 30 lakes situated along the proposed Mackenzie Gas Project pipeline route in the Canadian Northwest Territories were examined. Sampled lakes were shallow $\left(Z_{\max }=1.5-30 \mathrm{~m}\right.$; mean depth $\left.=6.6 \mathrm{~m}\right)$, generally nutrient poor (TP often $\leq 10 \mu \mathrm{g} \mathrm{L}^{-1}$ ), and alkaline (mean $\mathrm{pH}=8.2$ ). Floodplain lakes located within Arctic-tundra watersheds tended to have higher conductivity and major ions ( $\left.\mathrm{Na}, \mathrm{Cl}, \mathrm{Ca}, \mathrm{SO}_{4}\right)$ concentrations relative to non-flooded Arctic-tundra or forest-tundra lakes, reflecting differences in vegetation, elevation and most importantly, proximity to the Mackenzie River. The first two axes of a principal component analysis explained $56.1 \%$ of the variance in the environmental data. Variables most strongly associated with the first principal component axis were latitude, elevation, dissolved oxygen, temperature, conductivity and turbidity while the second principal component axis represented gradients of nutrients and Chlorophyll-a (Chl-a). Factors affecting Chl-a varied among the different ecological zones. This study provides reference data for future monitoring of potential effects of development and warming in the Arctic.
\end{abstract}

Key words: limnology, climate change, Mackenzie River basin, phytoplankton, nutrients, multivariate analyses

\section{INTRODUCTION}

The lower Mackenzie River basin (MRB) is a relatively isolated and undisturbed watershed located in the Canadian Northwest Territories (NT; MRBB 2004). However, this condition could change with the implementation of the Mackenzie Gas Project (MGP) with its three drill pads located in the Mackenzie River delta region and a pipeline running south $1220 \mathrm{~km}$ into northern Alberta (Imperial Oil Resources Ventures Limited 2004). Some of the activities related to drill pad and pipeline construction and operation that may have adverse effects on aquatic ecosystems include habitat disruption, water diversion and increased nutrient releases associated with sewage discharges (MRBB 2004). Although some limnological studies have been conducted in the general area of the proposed drill pads and pipeline route, they focused on lakes in the upper Mackenzie River delta (Squires \& Lesack 2003; Spears \& Lesack 2006), on Tuktoyaktuk Peninsula (Fee et al. 1988; Anema et al. 1990; Ramlal et al. 1991), or further south near Yellowknife, NT (Pienitz et al. 1997a, b). As well, baseline environmental impact assessment studies that were conducted as part of the MGP (Imperial Oil Resources Ventures Limited 2004), obtained very limited information on the water chemistry of lakes situated along the proposed pipeline route including Chlorophyll- $a$ (Chl- $a$ ) concentrations and phytoplankton and invertebrate communities (Evans et al. 2007). Conse- quently, there is a very limited understanding of the relationship between algal biomass (measured as Chl- $a$ ) and environmental factors in the lakes near the drill pad areas and along the pipeline route.

Since the mid-1960s, empirical models based on the regressions of Chl- $a$ on epilimnetic total phosphorus (TP) have been used to predict algal biomass in lakes (Sakamoto 1966; Schindler 1977; McCauley et al. 1989; Evans et al. 1996; Elser et al. 2007; Schindler et al. 2008). Such models have strong support from empirical studies (Dillon \& Rigler 1974), whole-lake experimental studies (Schindler 1977), and successful management of eutrophication (Edmondson 1991). However, because these empirically-derived models were based on data from northern temperate regions, they may not apply to subarctic and Arctic lakes (Flanagan et al. 2003).

Recent studies suggest that Arctic freshwaters respond differently to TP addition than temperate systems because they have very different chemical and physical properties (Rühland et al. 2003; Prowse et al. 2006). Moreover, dramatic changes in the prevailing environmental features of the Arctic (i.e., extreme seasonal variations in solar radiation and water temperatures, and persistently low nutrients) due to global climate change may have profound effects on phytoplankton biomass. Some of the effects associated with warming, such as reduced ice cover and thawing permafrost in combination with increased water temperatures 


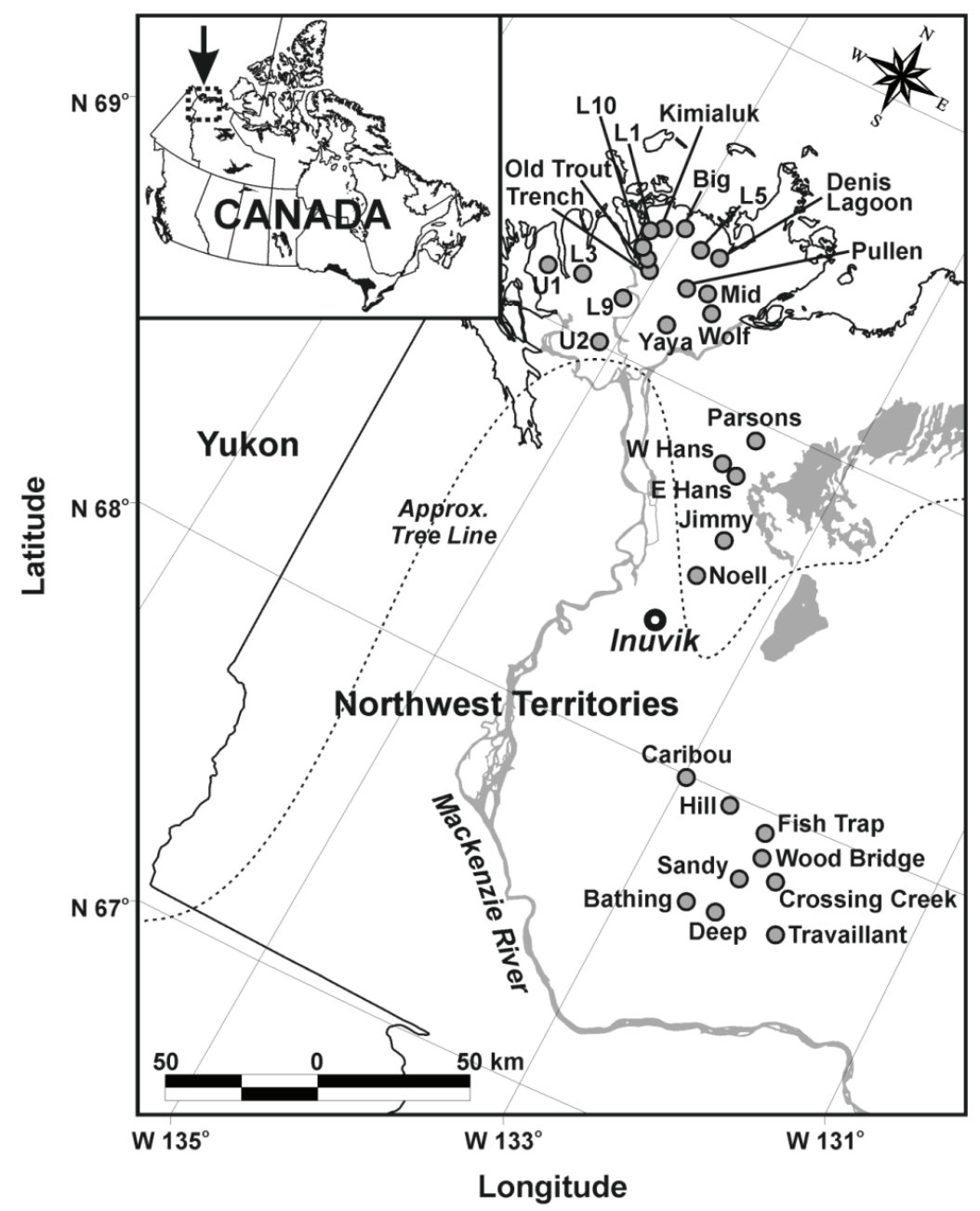

Fig. 1. Location of 30 study lakes in the lower Mackenzie River basin (inset) near Inuvik, Northwest Territories, Canada.

(ACIA 2005), may significantly enhance algal growth, productivity, and nutrient cycling (Flanagan et al. 2003). Similarly, allochthonous input of nutrients into lakes from surrounding catchments as a result of degraded permafrost may enhance primary production while high dissolved organic carbon (DOC) influx may actually attenuate light and limit phosphorus bioavailability for microbial production. The extent to which the above named environmental factors interact to affect phytoplankton biomass in high latitude lakes has not been systematically evaluated (Flanagan et al. 2003). Here we report on the water chemistry and the environmental factors controlling phytoplankton standing stocks using data obtained from surveys of 30 lakes carried out during ice-free open-water seasons in 2004, 2006 and 2007. These lakes are distributed along a north-south transect on the eastern side of the Mackenzie River and lie along a vegetational gradient of forest-tundra in the south to Arctic-tundra in the north (Fig. 1).

The primary objectives of this study were to (i) describe the physical and chemical characteristics of lakes situated along the proposed MGP drill pads and pipeline route and compare these findings with previous data published from the Mackenzie River delta and lakes in the Tuktoyatuk and Yellowknife area, (ii) examine the nutrients limiting algal standing stocks in these sites using sestonic and nutrient ratios, (iii) use principal component analysis (PCA) to identify lakes with common limnological features and the factors affecting these groupings, and (iv) use linear regressions and general linear models (GLM) to predict Chl- $a$. This investigation is part of a more extensive study conducted to acquire predisturbance baseline data (e.g., sediment, water chemistry, benthic macroinvertebrates, zooplankton, and phytoplankton standing stocks and diversity) of lakes situated near the proposed MGP production facilities and pipelines in the vicinity of Inuvik, NT. Results of the nutrient limitation bioassays and sestonic ratios have been published elsewhere (Ogbebo et al. 2009). It is our intention that the accrued limnological information will add to the growing reference database for long-term monitoring of the response of northern lakes to human impacts and/or climate change (Ramlal et al. 1991; Pienitz et al. 1997a, b; Rủhland et al. 2003). 


\section{MATERIALS AND METHODS}

\subsection{Study area and lake selection}

The 30 lakes selected for this study are situated in the lower MRB, and lie along a latitudinal gradient between $67^{\circ} 39^{\prime}$ and $69^{\circ} 24^{\prime} \mathrm{N}$ (Fig. 1). Sixteen of these sites are located in the Mackenzie River delta while the remaining 14 are situated along the eastern side of the Mackenzie River (Fig. 1). Lakes south of Inuvik are below the tree line and are situated in the forest-tundra ecological zone while those north are located in the Arctic-tundra ecological zone. Within the Arctic-tundra study area there were two groups of lakes: those that are situated at lower elevation ( $\leq 5 \mathrm{~m}$ above sea level (ASL)) and are predisposed to regular flooding by the Mackenzie River, and those that are not flooded $(\geq 5 \mathrm{~m}$ ASL; Tab. 1). Furthermore, the forest-tundra lakes sampled during this study are situated at higher elevations along the pipeline corridor and are not flooded by the Mackenzie River. These lakes represent a gradient in depth, elevation, DOC, nutrients, connectivity to the Mackenzie River, and proximity to locations of the proposed MGP production facilities and pipelines. One drill pad will be in the Big and Kimialuk lakes area (Taglu), a second in the Old Trout and Trench lakes area (Niglintgak), and the third at Parsons Lake.

\subsection{Regional climate and vegetation}

The climate and weather in this region are highly variable due to its close proximity to the Beaufort Sea. Inuvik has an intermediate climate between subarctic and Arctic with very long, cold and dark winters; there is continuous sunlight during the summer season. The average climatic conditions in this region are described in the Environment Canada website (Environment Canada 2005, Climate Normals 19712000).

Detailed information on the vegetation in the study area is presented in Ogbebo et al. (2009). Briefly, forest-tundra and Arctic-tundra comprise the main vegetation zones of the study area. The area north of Inuvik lies in the Arctic-tundra ecological zone and can be described as a vast area of barren landscape with permanently frozen subsoil (permafrost). It is mainly a treeless zone consisting of grasses, dwarfed shrubs, herbs, lichens, mosses and sedges. In contrast, the region to the south of Inuvik lies below the treeline and is classified as the subarctic forest-tundra ecological zone. The forest-tundra zone is characterized by forest patches which are dominated by Picea glauca (white spruce), $P$. mariana (black spruce) and Larix laricina (larch) with an understory of diverse sedges, mosses, lichens, grasses and herbs. Tree growth in the northern part of the transition zone is hampered by low precipitation and cold winters resulting in small-scattered clumps of stunted spruce, tamarack and dwarf birch (Betula nana) on warmer, sheltered sites (Imperial Oil
Resources Ventures Limited 2004). Additionally, vegetation and soil conditions in both ecological zones are strongly influenced by permafrost, which is continuous above the treeline and discontinuous below the treeline (Pienitz et al. 1997a, b). Catchment vegetation in the forest-tundra regions may be a significant source of carbon $(\mathrm{C})$ to lakes.

\subsection{Sampling protocol and analyses}

A total of 30 lakes were sampled for this study (Fig. 1). Eight lakes were sampled during 20-22 August 2004, 17 during 10-14 August 2006, and 17 lakes during 2327 July 2007; winter sampling was limited to one occasion on a small number of lakes and is reported in Ogbebo et al. (2009). Eight of the lakes were sampled on two occasions, and Parsons and East Hans lakes were sampled during all three time periods. Some of the lakes, such as Big and Parsons, were sampled more than once because of their proximity to drill pads and our anticipation of a long-term monitoring program occurring at or near (control) these lakes. Because of the similarity between data from multiple sampling, only the mean of the observations are used in this study.

All study lakes were accessible only by air, so sampling was conducted from a DHC-2 Beaver floatplane that landed near the centre of each lake. Measurements such as temperature, $\mathrm{pH}$, specific conductivity, dissolved oxygen (DO), and turbidity were made in situ using a YSI 6600 multiparameter sonde. Water transparency was determined using a standard 22-cm black and white Secchi disk. Surface ( $c a 0.5 \mathrm{~m}$ ) water samples were collected by immersing an acid-washed, pre-rinsed Nalgene bottle just below the surface, while deep samples were obtained with a Niskin water bottle at a depth of $1 \mathrm{~m}$ above the lake floor. All samples were immediately placed in coolers containing ice packs. Within $6 \mathrm{~h}$ of collection, the samples were filtered and prepared for transport to analytical laboratories for various analyses. Water was analyzed for major ions $\mathrm{Ca}, \mathrm{Mg}, \mathrm{K}, \mathrm{Na}, \mathrm{Cl}$, and $\mathrm{SO}_{4}$ ), total metals, dissolved inorganic carbon (DIC), DOC, and nutrients and related variables including total phosphorus (TP), dissolved phosphorus (DP), orthophosphate (ortho-P), particulate organic carbon (POC), particulate organic nitrogen (PN), total dissolved nitrogen (TDN), ammonia $\left(\mathrm{NH}_{3}\right)$, nitrate-nitrite $\left(\mathrm{NO}_{3}+\mathrm{NO}_{2}\right)$, and silica $\left(\mathrm{SiO}_{2}\right)$. Particulate phosphorus (PP) was estimated as the difference between TP and DP. Total nitrogen (TN) is the sum of TDN and PN. The above abbreviations in parentheses correspond to the labels used in the figures and tables. All analyses were carried out at the National Laboratory for Environmental Testing (NLET) in Saskatoon, SK and Burlington, $\mathrm{ON}$, and at the Prairie and Northern Laboratory for Environmental Testing (PNLET) in Edmonton, AB. All three laboratories followed standard protocols outlined by Environment Canada (1992). 
Tab. 1. The chemical and physical parameters for study lakes. Lat. = latitude; Long. = longitude; Elev. = elevation; Temp. = temperature; Cond. = specific conductivity; Turb. = turbidity; Secchi $=$ Secchi depth.

\begin{tabular}{|c|c|c|c|c|c|c|c|c|c|c|c|c|c|c|}
\hline Lake & $\begin{array}{l}\text { Lat. } \\
\text { (N) }\end{array}$ & $\begin{array}{l}\text { Long. } \\
\text { (W) }\end{array}$ & $\begin{array}{l}\text { Elev. } \\
(\mathrm{m})\end{array}$ & $\begin{array}{l}\text { Area } \\
\text { (ha) }\end{array}$ & $\begin{array}{l}\text { Depth } \\
\text { (m) }\end{array}$ & $\begin{array}{c}\mathrm{DO} \\
\left(\mathrm{mg} \mathrm{L}^{-1}\right)\end{array}$ & $\mathrm{pH}$ & $\begin{array}{l}\text { Temp. } \\
\left({ }^{\circ} \mathrm{C}\right)\end{array}$ & $\begin{array}{l}\text { Cond. } \\
\left(\mu \mathrm{S} \mathrm{cm}^{-1}\right)\end{array}$ & $\begin{array}{l}\text { Turb. } \\
\text { (NTU) }\end{array}$ & $\begin{array}{l}\text { Secchi } \\
(\mathrm{m})\end{array}$ & $\begin{array}{c}\mathrm{TP} \\
\left(\mu \mathrm{g} \mathrm{L}^{-1}\right)\end{array}$ & $\begin{array}{c}\mathrm{DP} \\
\left(\mu \mathrm{g} \mathrm{L}^{-1}\right)\end{array}$ & $\begin{array}{c}\mathrm{TDN} \\
\left(\mu \mathrm{g} \mathrm{L}^{-1}\right)\end{array}$ \\
\hline \multicolumn{15}{|l|}{ Forest-tundra } \\
\hline Bathing & $67^{\circ} 39^{\prime}$ & $132^{\circ} 27^{\prime}$ & 205 & 1974 & 5.8 & 9.7 & 8.5 & 16.1 & 169.0 & 0.3 & 5.5 & 2.5 & 2.5 & 245.0 \\
\hline Deep & $67^{\circ} 40^{\prime}$ & $132^{\circ} 15^{\prime}$ & 206 & 1413 & 44.0 & 9.4 & 8.3 & 15.7 & 122.0 & 0.2 & 9.0 & 2.5 & 2.5 & 155.0 \\
\hline Travaillant & $67^{\circ} 41^{\prime}$ & $131^{\circ} 41^{\prime}$ & 140 & 11321 & 5.7 & 9.6 & 8.3 & 17.1 & 118.7 & 0.6 & 5.2 & 2.3 & 1.8 & 318.0 \\
\hline Sandy & $67^{\circ} 48^{\prime}$ & $132^{\circ} 14^{\prime}$ & 161 & 2113 & 18.5 & 9.6 & 8.2 & 15.1 & 97.0 & 0.3 & 3.5 & 2.5 & 2.5 & 385.0 \\
\hline Crossing Creek & $67^{\circ} 49^{\prime}$ & $132^{\circ} 01^{\prime}$ & 148 & 272 & 7.5 & 9.7 & 8.1 & 17.0 & 97.5 & 1.5 & 2.3 & 4.5 & 1.0 & 388.0 \\
\hline Wood Bridge & $67^{\circ} 52^{\prime}$ & $132^{\circ} 09^{\prime}$ & 174 & 478 & 8.7 & 9.1 & 7.9 & 17.3 & 83.0 & 0.6 & 2.3 & 5.5 & 1.0 & 456.5 \\
\hline Fish Trap & $67^{\circ} 56^{\prime}$ & $132^{\circ} 13^{\prime}$ & 226 & 324 & 13.9 & 9.3 & 7.8 & 16.8 & 80.0 & 2.4 & 2.3 & 12.5 & 3.0 & 522.5 \\
\hline Hill & $67^{\circ} 58^{\prime}$ & $132^{\circ} 32^{\prime}$ & 226 & 811 & 1.8 & 10.4 & 8.3 & 16.2 & 75.0 & 5.4 & 0.8 & 38.5 & 5.0 & 500.5 \\
\hline Caribou & $67^{\circ} 59^{\prime}$ & $132^{\circ} 93^{\prime}$ & 197 & 5455 & 4.7 & 9.4 & 8.1 & 16.3 & 64.3 & 1.3 & 2.1 & 5.0 & 2.6 & 292.5 \\
\hline MIN & $67^{\circ} 39^{\prime}$ & $132^{\circ} 27^{\prime}$ & 140 & 272 & 1.8 & 9.1 & 7.8 & 15.1 & 64.3 & 0.2 & 0.8 & 2.3 & 1.0 & 155.0 \\
\hline MAX & $67^{\circ} 59^{\prime}$ & $132^{\circ} 32^{\prime}$ & 226 & 11321 & 44.0 & 10.4 & 8.5 & 17.3 & 169.0 & 5.4 & 9.0 & 38.5 & 5.0 & 522.5 \\
\hline MEAN & & & 187 & 2684 & 10.7 & 9.6 & 8.2 & 16.4 & 100.7 & 1.4 & 3.6 & 8.4 & 2.4 & 362.6 \\
\hline \multicolumn{15}{|c|}{ Arctic-tundra (non-flooded) } \\
\hline Noell & $68^{\circ} 31^{\prime}$ & $133^{\circ} 32^{\prime}$ & 87 & 3005 & 2.8 & 9.8 & 8.2 & 15.7 & 67.3 & 0.5 & 2.8 & 7.8 & 1.8 & 192.8 \\
\hline Jimmy & $68^{\circ} 38^{\prime}$ & $133^{\circ} 30^{\prime}$ & 48 & 1076 & 8.0 & 9.3 & 7.9 & 16.7 & 68.0 & 1.1 & 2.7 & 11.5 & 4.5 & 321.5 \\
\hline East Hans & $68^{\circ} 50^{\prime}$ & $133^{\circ} 39^{\prime}$ & 40 & 368 & 1.8 & 10.9 & 7.8 & 13.9 & 82.3 & 5.8 & 0.5 & 36.4 & 5.7 & 366.3 \\
\hline West Hans & $68^{\circ} 51^{\prime}$ & $133^{\circ} 32^{\prime}$ & 91 & 10 & 1.5 & 10.8 & 8.1 & 16.0 & 63.5 & 2.1 & 1.5 & 25.6 & 9.4 & 356.8 \\
\hline Parsons & $68^{\circ} 57^{\prime}$ & $133^{\circ} 39^{\prime}$ & 44 & 6349 & 3.7 & 10.4 & 7.8 & 15.0 & 66.9 & 2.9 & 1.7 & 22.0 & 4.3 & 398.3 \\
\hline Wolf & $69^{\circ} 13^{\prime}$ & $134^{\circ} 26^{\prime}$ & 27 & 972 & 5.0 & 10.4 & 8.3 & 15.2 & 179.0 & 0.6 & 3.5 & 3.0 & 1.0 & 174.0 \\
\hline Pullen & $69^{\circ} 14^{\prime}$ & $134^{\circ} 40^{\prime}$ & 27 & 711 & 7.5 & 10.3 & 8.4 & 14.5 & 117.5 & 0.5 & 2.7 & 5.5 & 1.0 & 240.5 \\
\hline Mid & $69^{\circ} 15^{\prime}$ & $134^{\circ} 32^{\prime}$ & 23 & 18 & 3.5 & 10.7 & 8.3 & 13.8 & 120.5 & 0.8 & 3.4 & 7.0 & 1.8 & 300.0 \\
\hline L5 & $69^{\circ} 21^{\prime}$ & $134^{\circ} 49^{\prime}$ & 9 & 150 & 7.8 & 9.5 & 8.1 & 14.9 & 318.7 & 12.2 & 0.9 & 28.3 & 5.0 & 400.0 \\
\hline MIN & $68^{\circ} 31^{\prime}$ & $133^{\circ} 32^{\prime}$ & 9 & 10.0 & 1.5 & 9.3 & 7.8 & 13.8 & 63.5 & 0.5 & 0.5 & 3.0 & 1.0 & 174.0 \\
\hline MAX & $69^{\circ} 21^{\prime}$ & $134^{\circ} 49^{\prime}$ & 91 & 6349.0 & 8.0 & 10.9 & 8.4 & 16.7 & 318.7 & 12.2 & 3.5 & 36.4 & 9.4 & 400.0 \\
\hline MEAN & & & 44 & 1406.6 & 4.6 & 10.2 & 8.1 & 15.1 & 120.4 & 2.9 & 2.2 & 16.3 & 3.8 & 305.6 \\
\hline \multicolumn{15}{|c|}{ Arctic-tundra (flooded) } \\
\hline $\mathrm{U} 2$ & $68^{\circ} 59^{\prime}$ & $135^{\circ} 23^{\prime}$ & 0 & 665 & 1.8 & 10.4 & 8.4 & 14.5 & 255.0 & 64.7 & 0.3 & 14.0 & 2.5 & 225.0 \\
\hline U1 & $69^{\circ} 07^{\prime}$ & $135^{\circ} 48^{\prime}$ & 0 & 665 & 1.8 & 10.7 & 8.4 & 14.9 & 235.0 & 16.8 & 0.5 & 2.5 & 2.5 & 190.0 \\
\hline L3 & $69^{\circ} 09^{\prime}$ & $13530^{\prime}$ & 0 & 31 & 2.0 & 10.6 & 8.2 & 15.9 & 342.3 & 10.2 & 0.4 & 36.7 & 5.0 & 100.0 \\
\hline L9 & $69^{\circ} 09^{\prime}$ & $135^{\circ} 10^{\prime}$ & 0 & 364 & 2.0 & 9.3 & 8.2 & 15.7 & 270.7 & 16.1 & 0.4 & 40.0 & 5.0 & 566.7 \\
\hline Yaya & $69^{\circ} 10^{\prime}$ & $134^{\circ} 37^{\prime}$ & 2 & 1034 & 28.0 & 10.5 & 8.4 & 14.1 & 291.0 & 0.7 & 4.2 & 2.3 & 1.8 & 209.0 \\
\hline Trench & $69^{\circ} 16^{\prime}$ & $135^{\circ} 06^{\prime}$ & 0 & 762 & 2.0 & 10.8 & 8.4 & 12.9 & 267.0 & 21.2 & 0.4 & 9.0 & 2.5 & 185.0 \\
\hline Old Trout & $69^{\circ} 17^{\prime}$ & $135^{\circ} 08^{\prime}$ & 0 & 740 & 10.7 & 10.5 & 8.5 & 13.9 & 297.0 & 1.6 & 2.7 & 2.5 & 2.5 & 160.0 \\
\hline L10 & $69^{\circ} 18^{\prime}$ & $135^{\circ} 11^{\prime}$ & 1 & 239 & 3.5 & 10.5 & 8.2 & 14.8 & 287.2 & 8.3 & 0.9 & 20.0 & 5.0 & 1080.0 \\
\hline L1 & $69^{\circ} 21^{\prime}$ & $135^{\circ} 12^{\prime}$ & 1 & 168 & 2.5 & 9.7 & 8.1 & 14.9 & 288.8 & 17.3 & 0.5 & 40.0 & 5.0 & 100.0 \\
\hline Kimialuk & $69^{\circ} 22^{\prime}$ & $135^{\circ} 08^{\prime}$ & 0 & 2418 & 1.9 & 10.6 & 8.3 & 14.0 & 262.0 & 42.0 & 0.2 & 41.5 & 4.8 & 186.5 \\
\hline Denis Lagoon & $69^{\circ} 22^{\prime}$ & $134^{\circ} 39^{\prime}$ & 1 & 18 & 3.0 & 10.2 & 8.4 & 13.4 & 286.0 & 20.0 & 0.5 & 9.0 & 2.5 & 160.0 \\
\hline Big & $69^{\circ} 24^{\prime}$ & $134^{\circ} 32^{\prime}$ & 2.2 & 1213 & 3.3 & 10.5 & 8.5 & 14.2 & 245.3 & 2.6 & 2.2 & 6.5 & 2.3 & 207.8 \\
\hline MIN & $68^{\circ} 59^{\prime}$ & $135^{\circ} 23^{\prime}$ & 0 & 18.0 & 1.8 & 9.3 & 8.1 & 12.9 & 235.0 & 0.7 & 0.2 & 2.3 & 1.8 & 100.0 \\
\hline MAX & $69^{\circ} 24^{\prime}$ & $134^{\circ} 32^{\prime}$ & 2.2 & 2418.0 & 28.0 & 10.8 & 8.5 & 15.9 & 342.3 & 64.7 & 4.2 & 41.5 & 5.0 & 1080.0 \\
\hline MEAN & & & 0.6 & 693.1 & 5.2 & 10.4 & 8.3 & 14.4 & 277.3 & 18.4 & 1.1 & 18.7 & 3.5 & 280.8 \\
\hline
\end{tabular}


Tab. 1. (continued).

\begin{tabular}{|c|c|c|c|c|c|c|c|c|c|c|c|c|c|c|}
\hline Lake & $\begin{array}{c}\mathrm{NH}_{3} \\
\left(\mu \mathrm{g} \mathrm{L}^{-1}\right)\end{array}$ & $\begin{array}{c}\mathrm{NO}_{3}+\mathrm{NO}_{2} \\
\left(\mu \mathrm{g} \mathrm{L}^{-1}\right)\end{array}$ & $\begin{array}{c}\text { POC } \\
\left(\mu \mathrm{g} \mathrm{L}^{-1}\right)\end{array}$ & $\begin{array}{c}\mathrm{PN} \\
\left(\mu \mathrm{g} \mathrm{L}^{-1}\right)\end{array}$ & $\begin{array}{c}\mathrm{PP} \\
\left(\mu \mathrm{g} \mathrm{L}^{-1}\right)\end{array}$ & $\begin{array}{c}\mathrm{TN} \\
\left(\mu \mathrm{g} \mathrm{L}^{-1}\right)\end{array}$ & $\begin{array}{c}\mathrm{DOC} \\
\left(\mathrm{mg} \mathrm{L}^{-1}\right)\end{array}$ & $\begin{array}{c}\text { DIC } \\
\left(\mathrm{mg} \mathrm{L}^{-1}\right)\end{array}$ & $\begin{array}{c}\text { Chl- } a \\
\left(\mu \mathrm{g} \mathrm{L}^{-1}\right)\end{array}$ & $\begin{array}{c}\mathrm{Cl} \\
\left(\mathrm{mg} \mathrm{L}^{-1}\right)\end{array}$ & $\begin{array}{c}\mathrm{SO}_{4} \\
\left(\mathrm{mg} \mathrm{L}^{-1}\right)\end{array}$ & $\begin{array}{c}\mathrm{Ca} \\
\left(\mathrm{mg} \mathrm{L}^{-1}\right)\end{array}$ & $\begin{array}{c}\mathrm{Na} \\
\left(\mathrm{mg} \mathrm{L}^{-1}\right)\end{array}$ & $\begin{array}{c}\mathrm{SiO}_{2} \\
\left(\mathrm{mg} \mathrm{L}^{-1}\right)\end{array}$ \\
\hline \multicolumn{15}{|l|}{ Forest-tundra } \\
\hline Bathing & 6.0 & 5.0 & 152.5 & 30.0 & 5.0 & 275.0 & 9.5 & 18.4 & 1.5 & 3.2 & 7.4 & 24.2 & 3.2 & 1.3 \\
\hline Deep & 2.5 & 5.0 & 5.0 & 224.0 & 5.0 & 379.0 & 4.0 & 14.2 & 1.3 & 1.1 & 4.3 & 17.6 & 1.7 & 1.5 \\
\hline Travaillant & 11.3 & 5.0 & 149.5 & 118.0 & 0.5 & 436.0 & 10.6 & 11.6 & 1.7 & 2.1 & 7.1 & 14.8 & 3.1 & 1.5 \\
\hline Sandy & 23.5 & 5.0 & 5.0 & 226.5 & 5.0 & 611.5 & 10.5 & 8.3 & 1.3 & 1.8 & 9.7 & 11.4 & 3.3 & 1.5 \\
\hline Crossing Creek & 18.0 & 23.5 & 203.5 & 182.0 & 3.5 & 570.0 & 10.5 & 8.5 & 2.3 & 2.0 & 8.2 & 11.9 & 3.1 & 1.5 \\
\hline Wood Bridge & 14.5 & 5.0 & 244.0 & 198.0 & 4.5 & 654.5 & 14.0 & 7.5 & 3.1 & 2.8 & 2.8 & 11.0 & 2.3 & 1.0 \\
\hline Fish Trap & 41.5 & 19.5 & 333.0 & 199.0 & 9.5 & 721.5 & 13.7 & 7.5 & 3.3 & 3.0 & 2.9 & 10.5 & 2.6 & 1.1 \\
\hline Hill & 14.0 & 5.0 & 3905.0 & 858.0 & 33.5 & 1358.5 & 11.1 & 7.5 & 26.4 & 2.1 & 0.9 & 9.3 & 2.5 & 0.7 \\
\hline Caribou & 8.0 & 5.0 & 448.5 & 236.0 & 3.3 & 528.5 & 7.7 & 6.0 & 5.9 & 2.2 & 2.5 & 7.3 & 2.9 & 0.7 \\
\hline MIN & 2.5 & 5.0 & 5.0 & 30.0 & 0.5 & 275.0 & 4.0 & 6.0 & 1.3 & 1.1 & 0.9 & 7.3 & 1.7 & 0.7 \\
\hline MAX & 41.5 & 23.5 & 3905.0 & 858.0 & 33.5 & 1358.5 & 14.0 & 18.4 & 26.4 & 3.2 & 9.7 & 24.2 & 3.3 & 1.5 \\
\hline MEAN & 15.5 & 8.7 & 605.1 & 252.4 & 6.1 & 614.9 & 10.2 & 9.9 & 5.2 & 2.2 & 5.1 & 13.1 & 2.7 & 1.2 \\
\hline \multicolumn{15}{|c|}{ Arctic-tundra (non-flooded) } \\
\hline Noell & 6.3 & 5.0 & 103.0 & 8.8 & 6.0 & 201.5 & 4.9 & 4.3 & 1.6 & 1.7 & 11.0 & 7.1 & 2.5 & 0.4 \\
\hline Jimmy & 13.5 & 5.0 & 431.5 & 49.5 & 7.0 & 371.0 & 10.5 & 3.7 & 5.2 & 1.6 & 12.0 & 7.2 & 2.6 & 1.1 \\
\hline East Hans & 48.7 & 3.9 & 1721.4 & 275.9 & 30.7 & 642.1 & 11.4 & 6.2 & 14.9 & 3.2 & 8.1 & 10.0 & 2.2 & 2.3 \\
\hline West Hans & 50.0 & 6.0 & 770.6 & 165.0 & 16.2 & 521.8 & 11.7 & 5.2 & 4.6 & 2.7 & 2.1 & 7.6 & 1.9 & 1.3 \\
\hline Parsons & 58.0 & 5.3 & 1314.3 & 218.0 & 17.7 & 616.3 & 6.3 & 5.3 & 8.0 & 3.8 & 4.9 & 7.1 & 2.2 & 0.3 \\
\hline Wolf & 8.0 & 5.0 & 162.5 & 131.0 & 2.0 & 305.0 & 3.6 & 15.2 & 2.2 & 10.1 & 11.7 & 22.0 & 5.8 & 0.2 \\
\hline Pullen & 9.5 & 5.0 & 308.5 & 172.5 & 4.5 & 413.0 & 6.2 & 7.7 & 3.1 & 10.8 & 5.0 & 11.6 & 6.4 & 0.5 \\
\hline Mid & 9.3 & 5.0 & 484.0 & 131.0 & 5.3 & 431.0 & 4.3 & 10.6 & 4.5 & 9.4 & 3.6 & 13.0 & 5.4 & 0.3 \\
\hline L5 & 20.0 & 5.7 & 567.7 & 79.3 & 23.3 & 479.3 & 17.5 & nd & 2.5 & 11.7 & 37.8 & 39.0 & 9.6 & 3.2 \\
\hline MIN & 6.3 & 3.9 & 103.0 & 8.8 & 2.0 & 201.5 & 3.6 & 3.7 & 1.6 & 1.6 & 2.1 & 7.1 & 1.9 & 0.2 \\
\hline MAX & 58.0 & 6.0 & 1721.4 & 357.0 & 30.7 & 642.1 & 17.5 & 22.5 & 14.9 & 11.7 & 37.8 & 39.0 & 9.6 & 3.2 \\
\hline MEAN & 23.1 & 5.1 & 618.0 & 158.8 & 11.9 & 452.3 & 8.4 & 9.0 & 5.0 & 6.3 & 13.4 & 16.1 & 4.6 & 1.3 \\
\hline \multicolumn{15}{|c|}{ Arctic-tundra (flooded) } \\
\hline U2 & 12.5 & 55.0 & 874.0 & 124.5 & 11.5 & 349.5 & 6.5 & 21.9 & 5.5 & 5.1 & 38.1 & 35.8 & 5.8 & 3.5 \\
\hline U1 & 8.5 & 5.0 & 418.5 & 59.0 & 0 & 249.0 & 5.5 & 20.1 & 2.9 & 11.2 & 25.2 & 31.3 & 8.2 & 2.3 \\
\hline L3 & 20.0 & 7.3 & 1003.0 & 110.0 & 31.7 & 210.0 & 20.6 & nd & 3.1 & 23.8 & 37.8 & 40.6 & 16.2 & 3.1 \\
\hline L9 & 53.3 & 4.3 & 1096.7 & 111.3 & 35.0 & 678.0 & 18.2 & nd & 3.8 & 9.4 & 37.0 & 37.2 & 8.3 & 2.7 \\
\hline Yaya & 11.8 & 13.5 & 97.3 & 75.5 & 0.5 & 284.5 & 3.9 & 24.4 & 1.4 & 11.6 & 37.7 & 38.8 & 9.7 & 3.3 \\
\hline Trench & 8.0 & 5.0 & 316.0 & 357.0 & 6.5 & 542.0 & 8.0 & 22.5 & 3.5 & 7.9 & 37.7 & 36.5 & 7.4 & 3.1 \\
\hline Old Trout & 5.5 & 5.0 & 134.5 & 336.0 & 0 & 496.0 & 7.5 & 24.9 & 1.8 & 10.4 & 41.4 & 40.3 & 9.2 & 3.1 \\
\hline L0 & 20.0 & 4.1 & 542.4 & 84.6 & 15.0 & 1164.6 & 17.5 & nd & 2.6 & 7.6 & 29.2 & 31.1 & 6.4 & 2.6 \\
\hline L1 & 20.0 & 9.0 & 709.5 & 88.5 & 35.0 & 188.5 & 18.5 & nd & 2.9 & 14.5 & 39.1 & 39.7 & 11.2 & 3.3 \\
\hline Kimialuk & 12.3 & 27.5 & 641.3 & 117.8 & 36.8 & 304.3 & 6.9 & 21.9 & 6.4 & 6.1 & 36.7 & 35.3 & 6.2 & 3.2 \\
\hline Denis Lagoon & 7.5 & 5.0 & 263.0 & 42.0 & 6.5 & 202.0 & 9.0 & 21.5 & 3.9 & 16.2 & 36.6 & 35.3 & 11.6 & 2.6 \\
\hline Big & 8.8 & 5.0 & 304.0 & 58.5 & 4.3 & 266.3 & 6.6 & 23.0 & 3.0 & 14.5 & 17.5 & 33.6 & 9.2 & 1.3 \\
\hline MIN & 5.5 & 4.1 & 97.3 & 42.0 & 0.0 & 188.5 & 3.9 & 20.1 & 1.4 & 5.1 & 17.5 & 31.1 & 5.8 & 1.3 \\
\hline MAX & 53.3 & 55.0 & 1096.7 & 357.0 & 36.8 & 1164.6 & 20.6 & 24.9 & 6.4 & 23.8 & 41.4 & 40.6 & 16.2 & 3.5 \\
\hline MEAN & 15.7 & 12.2 & 533.3 & 130.4 & 15.2 & 411.2 & 10.7 & 22.5 & 3.4 & 11.5 & 34.5 & 36.3 & 9.1 & 2.9 \\
\hline
\end{tabular}


Phytoplankton biomass was measured as Chl- $a$ according to the method of Nusch (1980). Aliquots of water (500-1000 $\mathrm{mL}$; depending on lake productivity) were filtered through $47 \mathrm{~mm} \mathrm{GF} / \mathrm{C}$ filters. The filter was placed in a $15 \mathrm{~mL}$ centrifuge tube containing $10 \mathrm{~mL}$ of $90 \%$ ethanol, and the tube then wrapped in tin foil and stored at $-20{ }^{\circ} \mathrm{C}$ until extraction. Chl- $a$ was extracted from the filter by warming the sample tube in an $80^{\circ} \mathrm{C}$ water bath for approx. 5 min. Following removal from the water bath, the sample extract was cooled to room temperature and fluorescence determined using a Turner Designs fluorometer (Sunnyvale, CA, U.S.A.) equipped with narrow bandpass filters that screen out possible interference from pheopigments. Accordingly, Chl- $a$ values were not corrected for pheopigments.

\subsection{Statistical analyses}

Field data are reported as minimum, maximum, and mean for the three groups of lakes: forest-tundra, Arctic-tundra (non-flooded) and Arctic-tundra (flooded) (Tab. 1). For the lakes that stratify, Deep and Yaya, only measurements based on the surface sample are used; all other lakes include measurements from both depths sampled. Data include latitude, longitude, depth, DO, $\mathrm{pH}$, temperature, conductivity, turbidity, Secchi disk depth, TP, DP, PP, TN, TDN, $\mathrm{NO}_{3}+\mathrm{NO}_{2}, \mathrm{NH}_{3}, \mathrm{PN}$, DOC, DIC, Chl- $a$, POC, $\mathrm{SiO}_{2}, \mathrm{Ca}, \mathrm{Na}$, and $\mathrm{Cl}$. In cases where concentrations were below detection limits, half the detection limit was used to calculate the lake-season mean. All environmental variables, except $\mathrm{pH}$ (measured on a log-scale), latitude and longitude, were logtransformed to normalize their distribution prior to any statistical analyses.

Simple linear regression analyses were performed to determine the relationship between Chl- $a$ and nutrients (TP and TN) for all sites and for the three groups of lakes. A Pearson correlation matrix (PCM) was used to identify groups of significantly correlated environmental variables using Bonferroni's adjustment (Sokal \& Rohl 1995), taking average correlation among the variables into account (Sankoh et al. 1997). PCA, a multivariate ordination technique, was performed to show and interpret the major patterns of variation in the environmental data (Rühland et al. 2003). To reduce multicolinearity, some of the variables that were highly correlated based on the results of the PCM (above) were represented in the final PCA plot by proxy or a representative variable. For example, conductivity was used to represent all the major ions $\left(\mathrm{Ca}, \mathrm{Na}, \mathrm{Cl}\right.$, and $\left.\mathrm{SO}_{4}\right)$ and DIC; TP was used for POC, PP and DP; TN for TDN and PN; and turbidity represented Secchi disk depth (see table 2 for correlation coefficients among these variables). The exclusion of those variables resulted in 15 variables being included in the PCA that identified the major patterns of covariation within the dataset and ordinated the lakes with respect to environmental variables. Because several factors loaded high on more than one principal component in the PCA, we used varimax rotation to simplify the interpretation of the factor matrix and to limit confounding the analysis interpretation (Manly 1986). In the PCA correlation biplot, variables represented by long arrows have high variance and significant effects in axis (ter Braak \& Prentice 1988). Likewise, variables that are positively correlated often have small angles between their respective arrows (Pienitz et al. 1997a, b).

To better identify the potential nutrient responsible for limiting algal standing stocks in these lakes, several ratios including TN:TP (Smith 1982), sestonic C:N:P (Redfield 1958) and POC:Chl-a (Eppley et al. 1977) were calculated. The Redfield method uses sestonic ratios of $\mathrm{PC}, \mathrm{PN}$, and $\mathrm{PP}$ on a molar basis. $\mathrm{PC}: \mathrm{PN}$ ratios $>6.6$ are indicative of $\mathrm{N}$ deficiency, while $\mathrm{PC}: \mathrm{PP}$ ratios $>106$ and PN:PP $>16$ infer P deficiency. The Smith method is based on weights of TN and TP. Ratios $\geq 17$ infer that TP limits algal biomass while TN drives biomass at ratios $\leq 10 . \mathrm{N}$ and $\mathrm{P}$ co-limitation of algal growth is inferred when TN:TP ratios range from 10-17. POC:Chl- $a$ (wt:wt) were calculated to help elucidate whether $\mathrm{C}$ sources in these sites were mainly allochthonous $(\geq 100)$ or autochthonous ( $\leq 100$; Eppley et al. 1977).

A GLM was used to construct a model that best predicts Chl- $a$ concentration from other environmental variables. Initially, several variables including ecozone, water temperature, depth, turbidity, nutrients (TN and TP), DOC and conductivity were included in the GLM (see Smith 1982; Schindler 1977; Williamson et al. 1999). The best model was eventually determined through an iterative process of sequentially removing the most non-significant variable in the model until only statistically significant variables remained. Statistical analyses were performed using SYSTAT. For all analyses the level of significance was set at $p \leq 0.05$.

\section{RESULTS}

\subsection{Limnological characteristics}

The minimum, maximum and mean values of the limnological parameters are shown in table 1 . In general, most parameters exhibited a wide range in measured values. The lakes ranged in size from 10-11, 321 ha with elevation ranging from 0-226 $\mathrm{m}$ ASL. They were mostly shallow $(<10 \mathrm{~m}$ depth), with exceptions being Yaya Lake (44 m) and Deep Lake (30 m). Average summer surface (epilimnetic) water temperatures were moderate and decreased with increasing latitude from $17.3{ }^{\circ} \mathrm{C}$ at Wood Bridge Lake to $12.9^{\circ} \mathrm{C}$ at Trench Lake (Fig. 2a). Secchi disc depth ranged from $0.23 \mathrm{~m}$ at Kimialuk Lake, a shallow lake in the Mackenzie River delta, to $9 \mathrm{~m}$ at Deep Lake, and tended to decline with increasing latitude (Fig. 2b). Conductivity and major ion $\left(\mathrm{Na}, \mathrm{Cl}\right.$, and $\left.\mathrm{SO}_{4}\right)$ concentrations were greatest at higher latitudes, with the highest concentrations of these variables recorded in the flooded Arctic-tundra lakes, followed by non-flooded Arctic-tundra lakes and foresttundra lakes, respectively (Tab. 1). 
Tab. 2. Pearson correlation matrix for all variables (following transformations) measured in the 30 lakes included in the principal component analysys. Note: Values in bold are significant $(p \leq 0.0012)$ correlations. Lat. $=$ latitude; Elev. $=$ elevation; Secchi $=$ Secchi depth; Temp. $=$ temperature; Cond. $=$ specific conductivity; Turb. $=$ turbidity.

\begin{tabular}{|c|c|c|c|c|c|c|c|c|c|c|c|c|c|}
\hline & Lat. & Elev. & Depth & Area & DO & $\mathrm{pH}$ & Secchi & Temp. & Cond. & Turbid. & TP & DP & TDN \\
\hline Lat. & 1.00 & & & & & & & & & & & & \\
\hline Elev. & -0.84 & 1.00 & & & & & & & & & & & \\
\hline Depth & 0.33 & 0.30 & 1.00 & & & & & & & & & & \\
\hline Area & -0.25 & 0.14 & 0.18 & 1.00 & & & & & & & & & \\
\hline DO & 0.75 & -0.66 & -0.56 & -0.33 & 1.00 & & & & & & & & \\
\hline $\mathrm{pH}$ & 0.32 & -0.52 & -0.10 & 0.00 & 0.36 & 1.00 & & & & & & & \\
\hline Secchi & -0.46 & 0.59 & 0.71 & 0.21 & -0.53 & -0.05 & 1.00 & & & & & & \\
\hline Temp. & -0.83 & 0.79 & 0.23 & 0.24 & -0.77 & -0.47 & 0.46 & 1.00 & & & & & \\
\hline Cond. & 0.60 & -0.85 & -0.03 & -0.06 & 0.46 & 0.75 & -0.30 & -0.67 & 1.00 & & & & \\
\hline Turbid. & 0.51 & -0.68 & -0.67 & -0.20 & 0.53 & 0.12 & -0.96 & -0.47 & 0.42 & 1.00 & & & \\
\hline TP & 0.19 & -0.04 & -0.59 & -0.24 & 0.34 & -0.40 & -0.65 & -0.13 & -0.30 & 0.58 & 1.00 & & \\
\hline DP & 0.01 & -0.03 & -0.39 & -0.14 & 0.29 & -0.24 & -0.40 & -0.09 & -0.22 & 0.39 & 0.67 & 1.00 & \\
\hline TDN & -0.55 & 0.66 & 0.01 & -0.05 & -0.33 & -0.73 & 0.08 & 0.59 & -0.74 & -0.14 & 0.37 & 0.21 & 1.00 \\
\hline $\mathrm{NH}_{3}$ & -0.08 & 0.23 & -0.21 & -0.18 & 0.13 & -0.75 & -0.25 & 0.17 & -0.48 & 0.20 & 0.58 & 0.46 & 0.74 \\
\hline $\mathrm{NO}_{3}+\mathrm{NO}_{2}$ & 0.04 & -0.27 & 0.00 & -0.02 & 0.00 & -0.01 & -0.37 & 0.02 & 0.26 & 0.45 & 0.22 & -0.03 & 0.04 \\
\hline POC & 0.33 & -0.18 & -0.72 & -0.21 & 0.45 & -0.23 & -0.60 & -0.08 & -0.13 & 0.61 & 0.73 & 0.39 & 0.33 \\
\hline PN & -0.14 & 0.13 & 0.06 & -0.03 & 0.12 & -0.20 & -0.14 & -0.02 & -0.15 & 0.12 & 0.24 & 0.18 & 0.40 \\
\hline PP & 0.23 & -0.02 & -0.59 & -0.24 & 0.30 & -0.42 & -0.62 & -0.10 & -0.31 & 0.55 & 0.97 & 0.50 & 0.38 \\
\hline TN & -0.43 & 0.47 & 0.04 & 0.00 & -0.11 & -0.49 & -0.02 & 0.31 & -0.53 & -0.03 & 0.36 & 0.25 & 0.77 \\
\hline DOC & -0.49 & 0.36 & -0.14 & -0.12 & -0.35 & -0.46 & -0.18 & 0.43 & -0.40 & 0.13 & 0.34 & 0.33 & 0.68 \\
\hline DIC & 0.43 & $-\mathbf{- 0 . 7 3}$ & 0.01 & -0.02 & 0.40 & 0.76 & -0.22 & -0.58 & 0.97 & 0.35 & -0.35 & -0.21 & -0.67 \\
\hline Chl- $a$ & 0.17 & -0.02 & -0.61 & -0.17 & 0.35 & -0.34 & -0.62 & -0.10 & -0.29 & 0.56 & 0.86 & 0.57 & 0.42 \\
\hline $\mathrm{Cl}$ & 0.85 & -0.79 & -0.18 & -0.31 & 0.65 & 0.50 & -0.31 & -0.74 & 0.76 & 0.39 & -0.11 & -0.23 & -0.52 \\
\hline $\mathrm{SO}_{4}$ & 0.63 & -0.85 & -0.05 & 0.08 & 0.36 & 0.47 & -0.37 & -0.64 & 0.83 & 0.44 & -0.22 & -0.13 & -0.72 \\
\hline $\mathrm{Ca}$ & 0.52 & -0.81 & -0.02 & -0.04 & 0.42 & 0.73 & -0.29 & -0.62 & 0.99 & 0.42 & -0.30 & -0.19 & -0.71 \\
\hline $\mathrm{Na}$ & 0.76 & -0.85 & -0.08 & -0.15 & 0.51 & 0.68 & -0.30 & -0.72 & 0.88 & 0.41 & -0.27 & -0.30 & -0.64 \\
\hline $\mathrm{SiO}_{2}$ & 0.10 & -0.54 & -0.01 & -0.07 & 0.15 & 0.30 & -0.44 & -0.30 & 0.58 & 0.49 & -0.04 & 0.23 & -0.28 \\
\hline
\end{tabular}

Tab. 2. (continued).

\begin{tabular}{|c|c|c|c|c|c|c|c|c|c|c|c|c|c|c|}
\hline & $\mathrm{NH}_{3}$ & $\mathrm{NO}_{3}+\mathrm{NO}_{2}$ & POC & PN & PP & $\mathrm{TN}$ & DOC & DIC & Chl- $a$ & $\mathrm{Cl}$ & $\mathrm{SO}_{4}$ & $\mathrm{Ca}$ & $\mathrm{Na}$ & $\mathrm{SiO}_{2}$ \\
\hline \multicolumn{15}{|l|}{ Lat. } \\
\hline \multicolumn{15}{|l|}{ Elev. } \\
\hline \multicolumn{15}{|l|}{ Depth } \\
\hline \multicolumn{15}{|l|}{ Area } \\
\hline \multicolumn{15}{|l|}{ DO } \\
\hline \\
\hline \multicolumn{15}{|l|}{ Secchi } \\
\hline \multicolumn{15}{|l|}{ Temp. } \\
\hline \multicolumn{15}{|l|}{ Cond. } \\
\hline \multicolumn{15}{|l|}{ Turbid. } \\
\hline \multicolumn{15}{|l|}{$\mathrm{TP}$} \\
\hline \multicolumn{15}{|l|}{ DP } \\
\hline \multicolumn{15}{|l|}{ TDN } \\
\hline $\mathrm{NH}_{3}$ & 1.00 & & & & & & & & & & & & & \\
\hline $\mathrm{NO}_{3}+\mathrm{NO}_{2}$ & 0.18 & 1.00 & & & & & & & & & & & & \\
\hline POC & 0.45 & 0.13 & 1.00 & & & & & & & & & & & \\
\hline PN & 0.30 & 0.01 & 0.18 & 1.00 & & & & & & & & & & \\
\hline PP & 0.56 & 0.25 & 0.76 & 0.22 & 1.00 & & & & & & & & & \\
\hline $\mathrm{TN}$ & 0.52 & -0.04 & 0.28 & 0.85 & 0.34 & 1.00 & & & & & & & & \\
\hline DOC & 0.53 & 0.01 & 0.26 & 0.28 & 0.29 & 0.55 & 1.00 & & & & & & & \\
\hline DIC & -0.50 & 0.26 & -0.18 & -0.04 & -0.37 & -0.43 & -0.37 & 1.00 & & & & & & \\
\hline Chl- $a$ & 0.47 & 0.03 & 0.84 & 0.43 & 0.84 & 0.50 & 0.31 & -0.31 & 1.00 & & & & & \\
\hline $\mathrm{Cl}$ & -0.18 & 0.02 & 0.21 & -0.12 & -0.06 & -0.43 & -0.41 & 0.67 & -0.03 & 1.00 & & & & \\
\hline $\mathrm{SO}_{4}$ & -0.31 & 0.29 & -0.16 & -0.36 & -0.23 & -0.64 & -0.33 & 0.69 & -0.32 & 0.57 & 1.00 & & & \\
\hline $\mathrm{Ca}$ & -0.48 & 0.28 & -0.14 & -0.11 & -0.32 & -0.49 & -0.35 & 0.98 & -0.30 & 0.70 & 0.79 & 1.00 & & \\
\hline $\mathrm{Na}$ & -0.39 & 0.13 & 0.02 & -0.18 & -0.22 & -0.51 & -0.40 & 0.78 & -0.19 & 0.91 & 0.75 & 0.82 & 1.00 & \\
\hline $\mathrm{SiO}_{2}$ & -0.08 & 0.37 & -0.11 & 0.06 & -0.13 & -0.14 & 0.25 & 0.57 & -0.11 & 0.16 & 0.61 & 0.63 & 0.37 & 1.00 \\
\hline
\end{tabular}


Epilimnetic DO concentrations were high at all lakes ranging from 9.1-10.9 $\mathrm{mg} \mathrm{L}^{-1}$ while $\mathrm{pH}$ displayed a narrow range (7.8-8.5). The relatively deep lakes were well oxygenated; DO levels in both Deep and Yaya lakes were $>11 \mathrm{mg} \mathrm{L}^{-1}$ in the hypolimnion.
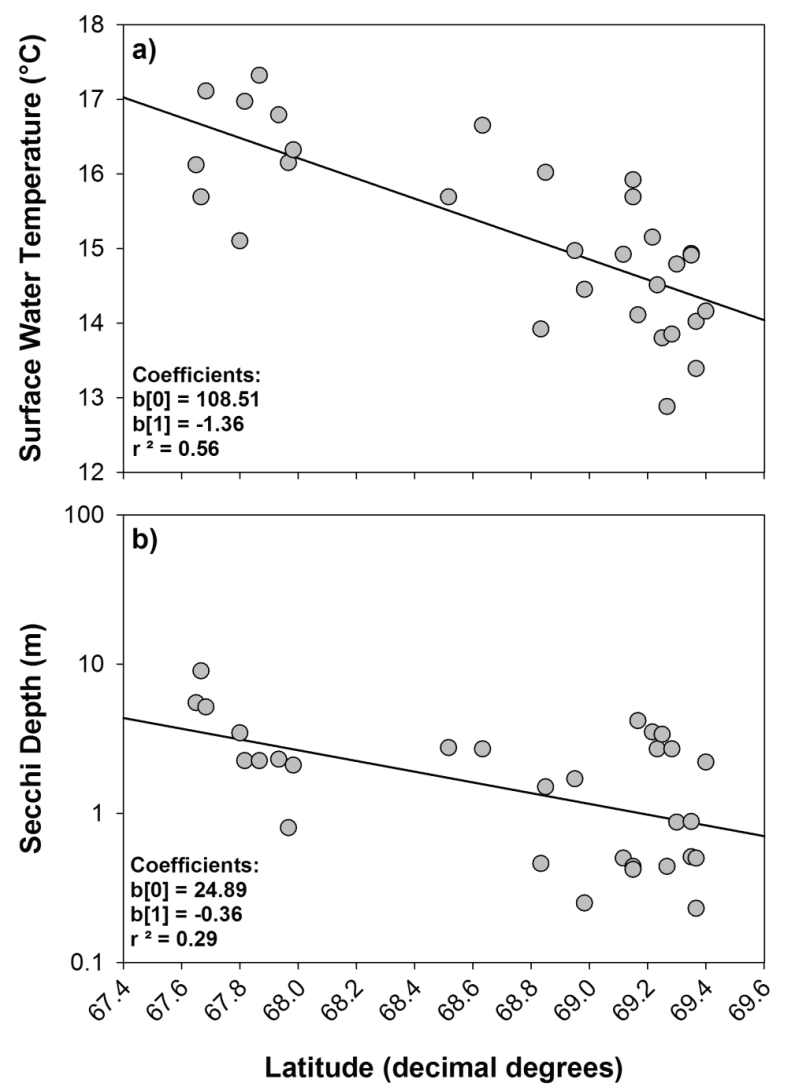

Fig. 2. Relationships between epilimnetic water temperature and water transparency with latitude for 30 lakes in the lower Mackenzie River basin, Northwest Territories, Canada.

TP concentrations were generally low $\left(\leq 10 \mu \mathrm{g} \mathrm{L}^{-1}\right)$, indicating the oligotrophic nature of these lakes, although there were instances of higher TP concentrations (36.4-41.0 $\mu \mathrm{g} \mathrm{L}^{-1}$ ), which would suggest mesotrophic condition in some lakes (Hill, East Hans, L3, L9, L1 and Kimialuk). In those lakes with high TP concentrations, DP was also low indicating that most of the phosphorus was in the particulate form (Tab. 1). Dissolved inorganic nutrients $\left(\mathrm{NO}_{3}+\mathrm{NO}_{2}, \mathrm{NH}_{3}, \mathrm{SiO}_{2}\right.$, and DP) were exceedingly low and often below detection limits; ortho-P was consistently below the detection limit at most lakes.

While there was no clear spatial pattern with regards to DOC concentrations, values were moderately high for all study lakes ranging from 3.6-20.6 $\mathrm{mg} \mathrm{L}^{-1}$ (mean = $9.9 \mathrm{mg} \mathrm{L}^{-1}$ ). DIC levels from study lakes were highly variable and ranged from $3.7-24.9 \mathrm{mg} \mathrm{L}^{-1}$ (mean $=13.1$ $\mathrm{mg} \mathrm{L}^{-1}$ ); relatively higher levels of DIC were observed at higher latitudes. Chl- $a$ concentrations were low $(\leq 6$ $\mu \mathrm{g} \mathrm{L}^{-1}$ ) with higher concentrations observed at Parsons, East Hans, Hill and Kimialuk lakes.

\subsection{Pearson correlation matrix}

The PCM of physical and chemical variables shows that many of the parameters were highly correlated (Tab. 2). DO and temperature were inversely related indicating that temperature was the main determinate of DO concentration. Environmental variables that express the ionic composition of water (i.e., conductivity, $\mathrm{Na}$, $\mathrm{Cl}, \mathrm{Ca}, \mathrm{SO}_{4}$, and DIC) were significant $(p \leq 0.0012)$ and highly intercorrelated (Tab. 2). In addition, they were negatively correlated with elevation, temperature, and TDN. DO, $\mathrm{Cl}, \mathrm{SO}_{4}$, and $\mathrm{Na}$ were positively and significantly $(p \leq 0.0012)$ correlated with latitude, while temperature, elevation and TDN were negatively correlated with latitude.

POC showed a strong positive correlation with Chl- $a$, turbidity and TP, while Chl- $a$ was positively correlated with TP, PP and POC, and negatively correlated with lake depth and Secchi depth. DOC was positively correlated with $\mathrm{pH}$, conductivity and TDN, while DIC was positively correlated with $\mathrm{pH}$, conductivity, and all the major ions.

\subsection{Nutrient limitation and sestonic ratios}

When nutrient deficiency was assessed from the total nutrient ratios, i.e., TN:TP, the mass ratio for most study lakes was $\geq 17$ (Fig. 3), strongly suggesting Plimitation. When assessed with PC:PN ratios (Fig. 4a), $\mathrm{N}$-limitation appeared to prevail in a majority of flooded Arctic-tundra lakes, while most forest-tundra lakes and non-flooded Arctic-tundra sites appeared to be N-sufficient. PC:PP ratios (Fig. 4b) and PN:PP ratios (Fig. 4c) indicated P-limitation in a majority of forest-tundra lakes and non-flooded Arctic-tundra lakes.

POC:Chl- $a$ ratios ranged from 3.7-326.5 (Fig. 5). Based on these ratios, nine lakes were likely autochthonous, nine likely intermediate, and 12 likely allochthonous.

\subsection{Principal components analysis}

The PCA biplot for the environmental variables is presented in figure $6 \mathrm{a}$. The variance in the environmental data explained by the first two principal component axes was $32.3 \%$ and $23.8 \%$, respectively. Only these two axes were retained for further interpretation because they explained more variation in the data than due to chance alone (Jackson 1993). Latitude, DO, conductivity, turbidity and $\mathrm{SiO}_{2}$ were positively associated with principal component one (PC1), while elevation, depth, water temperature and TN loaded negatively on PC1 (Tab. 3, Fig. 6a). For principal component two (PC2), depth loaded negatively, while TP, Chl- $a, \mathrm{NH}_{3}$, DOC, TN and turbidity loaded positively (Tab. 3, Fig. 6a). Thus, PC1 can be considered as a gradient of physical features, while PC2 can be identified primarily as a gradient of trophic state (nutrients and Chl-a). 


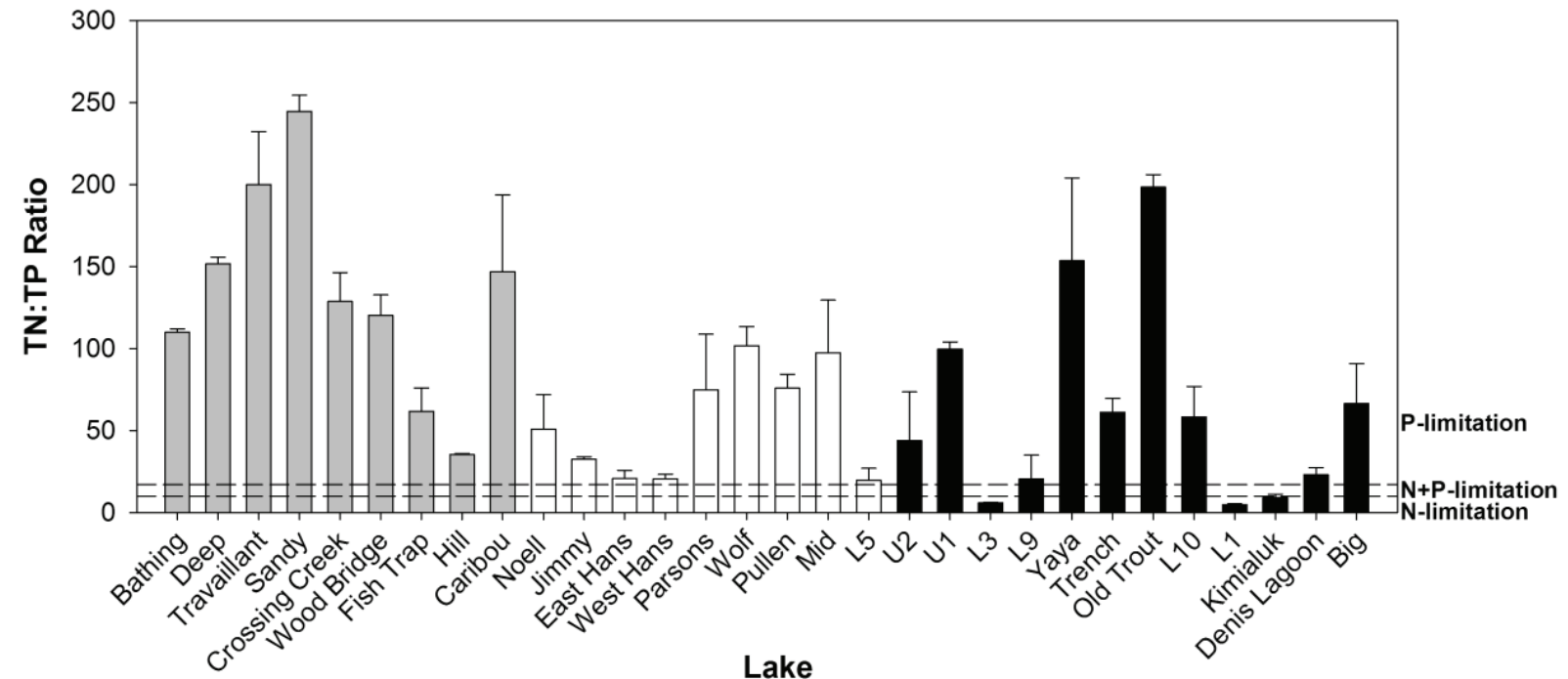

Fig. 3. Nutrient ratios (TN:TP) calculated from epilimnetic waters of study lakes. Data are means of three replicates ( \pm 1 SE). The horizontal reference lines represent nutrient ratios and boundaries for sufficiency or deficiency as outlined by Smith (1982). Lakes are arranged in order of increasing latitude. Grey bars = forest-tundra lakes; white bars = non-flooded Arctic-tundra lakes; black bars $=$ flooded Arctic-tundra lakes.
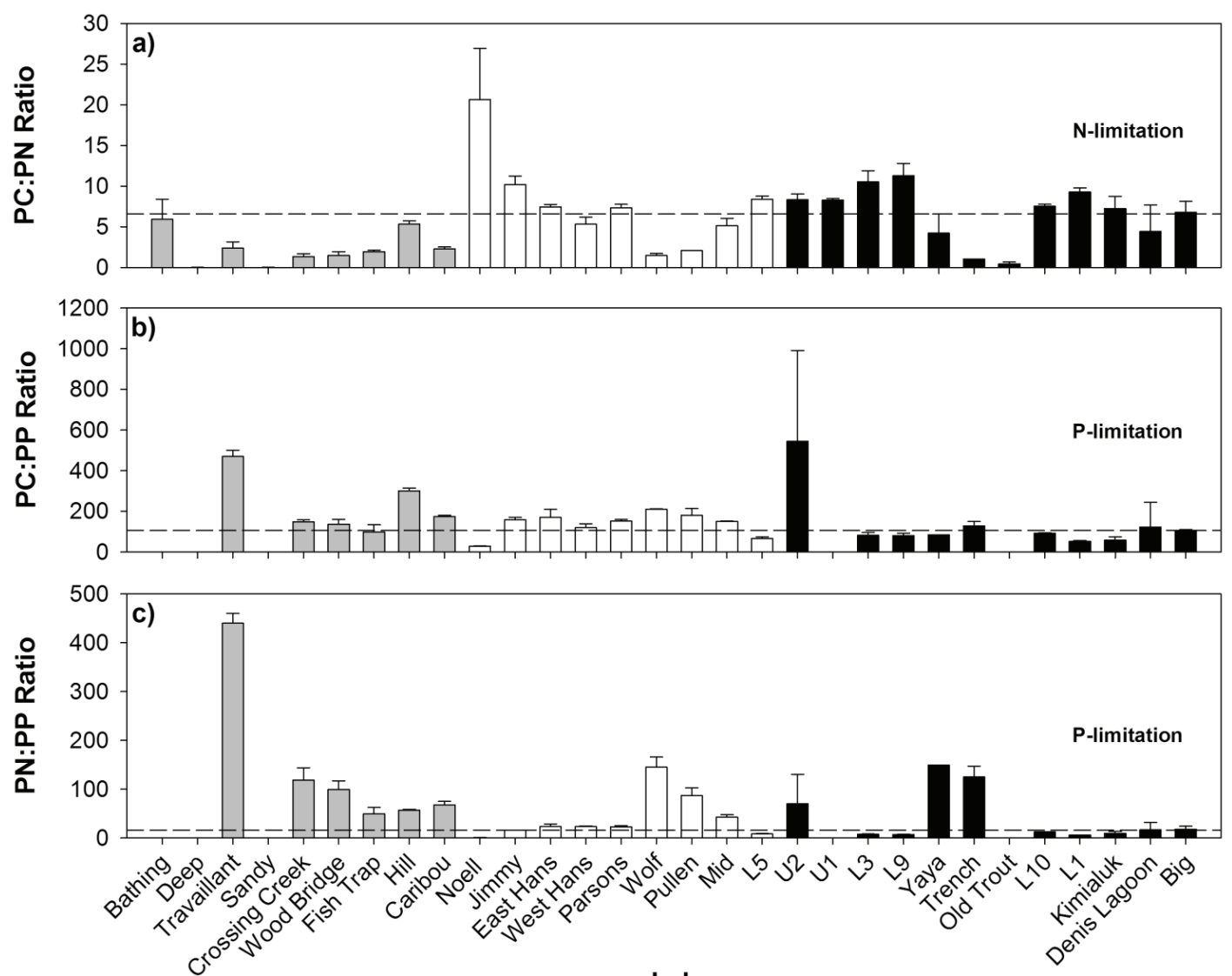

Lake

Fig. 4. Redfield sestonic ratios (PC:PN, PC:PP, and PN:PP) calculated from epilimnetic waters of study lakes. Data are means of three replicates $( \pm 1 \mathrm{SE})$. The horizontal reference lines represent nutrient ratios and boundaries for sufficiency or deficiency as outlined by Redfield (1958). Lakes are arranged in order of increasing latitude. Grey bars = forest-tundra lakes; white bars = nonflooded Arctic-tundra lakes; black bars = flooded Arctic-tundra lakes. 


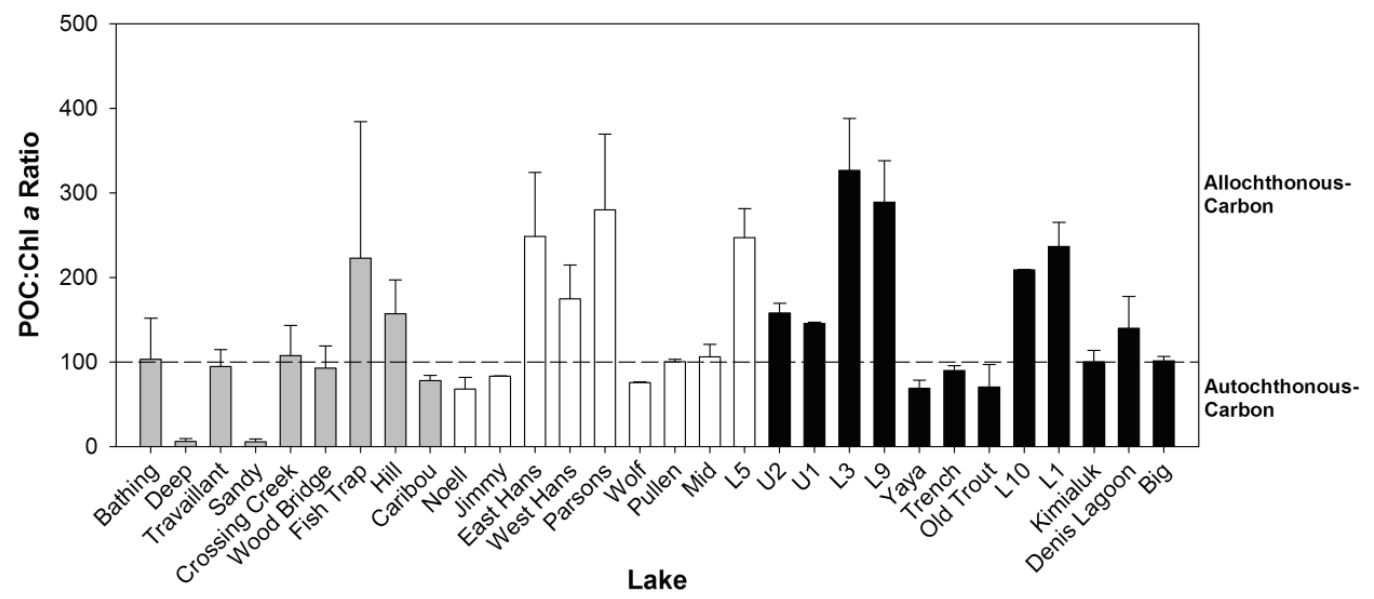

Fig. 5. POC:Chl- $a$ ratios determined from epilimnetic waters of study lakes. Data are means of three replicates $( \pm 1$ SE). The horizontal reference line defines carbon source. Lakes are arranged in order of increasing latitude. Grey bars = forest-tundra lakes; white bars = non-flooded Arctic-tundra lakes; black bars = flooded Arctic-tundra lakes.
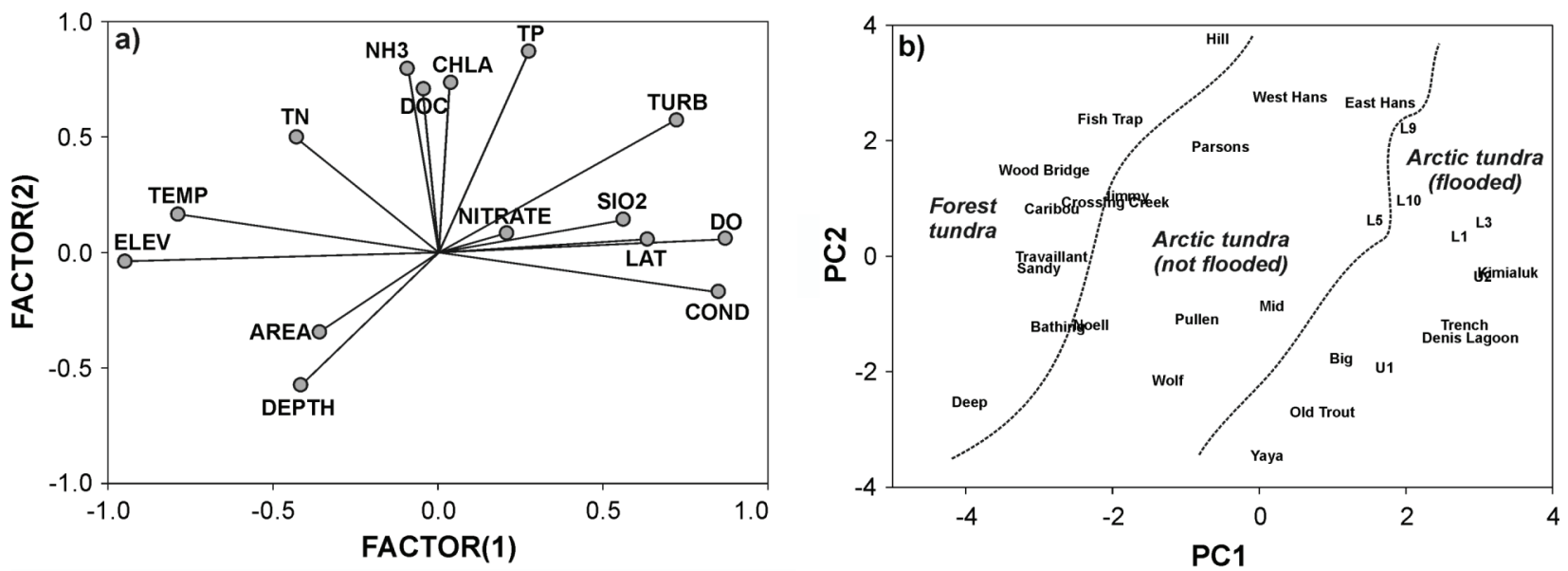

Fig. 6. (a) PCA of the physical, chemical and environmental variables from 30 lakes in the Northwest Territories, Canada. TEMP = temperature; ELEV = elevation; LAT = latitude; TURB = turbidity; COND = specific conductivity. (b) Ordination of the sample lakes in relation to their PCA scores from the physical, chemical and environmental variables; dotted lines are drawn by hand.

Lakes could be classified according to their respective ecological zone and proximity to the Mackenzie River delta (Tab. 1; Fig. 6b). Specifically, flooded Arctic-tundra lakes clustered on the right of the ordination along PC1 and were characterized as high latitude and low elevation, but were shallow, cold, and turbid lakes with high DO (probably due to wind-mixing) and conductivity. In contrast, forest-tundra lakes tended to cluster on the left along PC1 (Fig. 6b). Interestingly, the non-flooded Arctic-tundra lakes immediately north of Inuvik, being intermediate in location relative to the other two groups of lakes, were transitional in their limnological variables along PC1 (Fig. 6b). Small, shallow and turbid lakes with relatively higher nutrient and Chl$a$ concentrations all grouped together in the upper half of PC2, regardless of their vegetation or regional classification.
Tab. 3. Factor loadings after Varimax rotation for each limnological variable on the first two principal components. Values marked bold have considerable influence on the respective principal component.

\begin{tabular}{lcc}
\hline Variable & PC1 & PC2 \\
\hline Latitude & $\mathbf{0 . 8 6 9}$ & 0.060 \\
Elevation & $\mathbf{- 0 . 9 5 0}$ & -0.036 \\
Depth & $\mathbf{- 0 . 4 1 6}$ & $\mathbf{- 0 . 5 7 6}$ \\
Area & -0.360 & -0.346 \\
$\mathrm{DO}$ & $\mathbf{0 . 6 3 1}$ & 0.059 \\
Temperature & $\mathbf{- 0 . 7 8 9}$ & 0.167 \\
Conductivity & $\mathbf{0 . 8 4 6}$ & -0.169 \\
Turbidity & $\mathbf{0 . 7 2 2}$ & $\mathbf{0 . 5 7 4}$ \\
$\mathrm{TP}$ & 0.273 & $\mathbf{0 . 8 7 2}$ \\
$\mathrm{NH}_{3}$ & -0.093 & $\mathbf{0 . 7 9 9}$ \\
$\mathrm{NO}_{2}$-NO & 0.204 & 0.083 \\
$\mathrm{TN}$ & $\mathbf{- 0 . 4 3 1}$ & $\mathbf{0 . 5 0 1}$ \\
$\mathrm{DOC}$ & -0.046 & $\mathbf{0 . 7 1 1}$ \\
$\mathrm{Chl}^{-} a$ & 0.035 & $\mathbf{0 . 7 3 7}$ \\
$\mathrm{SiO}_{2}$ & $\mathbf{0 . 5 6 0}$ & 0.144 \\
\hline
\end{tabular}




\subsection{Models of Chl-a}

\subsubsection{Single variable-Chl- $a$ relationships}

To be consistent with previous reports of relationships between Chl- $a$ and nutrients (TP, TN), we report simple linear relationships for these variables. For all lakes, there was a highly significant positive relationship between TP and algal biomass $\left(\log _{10} \mathrm{Chl}-a=0.10\right.$ $+0.44 \log ^{10} \mathrm{TP} ; R^{2}=0.44, p \leq 0.0001$; Fig. 7a). The Chl$a$-TN relationship was positive and statistically significant for all 30 lakes $\left(\log _{10} \mathrm{Chl}-a=-0.95+0.56\right.$ $\log _{10} \mathrm{TN} ; R^{2}=0.16, p<0.05$; Fig. $7 \mathrm{~b}$ ), although considerably less variance in Chl- $a$ was explained by TN when compared to the TP-Chl- $a$ relationship.
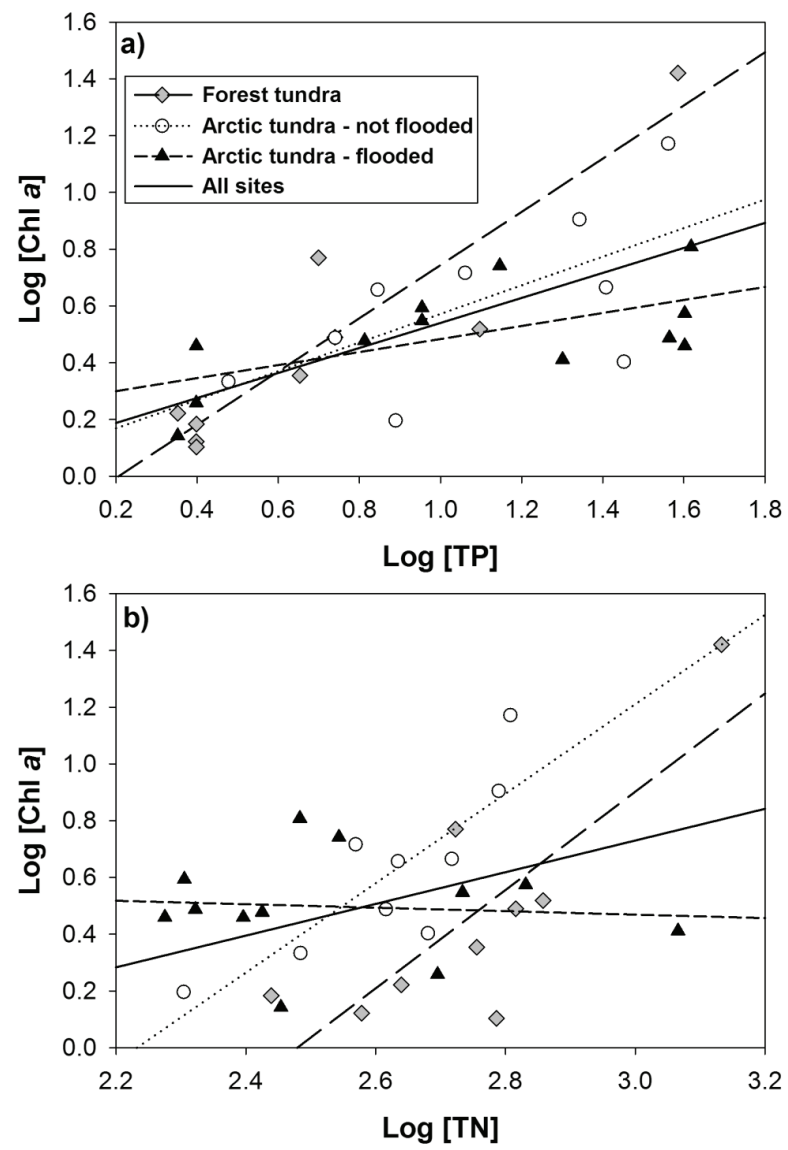

Fig. 7. (a) Plot of the linear regression between TP and Chl- $a$ concentrations for all lakes $\left(\log _{10}\right.$ Chl- $a=0.10+0.44 \log _{10} \mathrm{TP}$; $\left.R^{2}=0.44, p=0.0001\right)$, forest-tundra lakes $\left(\log _{10} \mathrm{Chl}-a=-0.19\right.$ $\left.+0.94 \log _{10} \mathrm{TP} ; R^{2}=0.84, p<0.0001\right)$, non-flooded Arctictundra lakes $\left(\log _{10} \mathrm{Chl}-a=0.07+0.50 \log _{10} \mathrm{TP} ; R^{2}=0.39, p=\right.$ 0.07 ), and flooded Arctic-tundra lakes ( $\log _{10} \mathrm{Chl}-a=0.25+$ $0.22 \log _{10} \mathrm{TP} ; R^{2}=0.38, p=0.03$ ). (b) Plot of the linear regression between $\mathrm{TN}$ and Chl- $a$ concentrations for all lakes $\left(\log _{10} \mathrm{Chl}-a=-0.95+0.56 \log _{10} \mathrm{TN} ; R^{2}=0.16, p=0.02\right)$, foresttundra lakes $\left(\log _{10} \mathrm{Chl}-a=-4.29+1.73 \log _{10} \mathrm{TN} ; R^{2}=0.64, p=\right.$ $0.01)$, non-flooded Arctic-tundra lakes $\left(\log _{10} \mathrm{Chl}-a=-3.52+\right.$ $1.58 \log _{10} \mathrm{TN} ; R^{2}=0.68, p=0.01$ ), and flooded Arctic-tundra lakes $\left(\log _{10} \mathrm{Chl}-a=0.65-0.06 \log _{10} \mathrm{TN} ; R^{2}=0.006, p=0.80\right)$.
Since the lakes tended to pattern out into three rather distinct types, we also calculated Chl- $a$-nutrient relationships for each unique lake type. A positive relationship was found between TP and Chl- $a$ for forest-tundra lakes $\left(\log _{10} \mathrm{Chl}-a=-0.19+0.94 \log _{10} \mathrm{TP} ; R^{2}=0.84, p\right.$ $<0.0001)$, flooded Arctic-tundra lakes $\left(\log _{10} \mathrm{Chl}-a=\right.$ $\left.0.25+0.22 \log _{10} \mathrm{TP} ; R^{2}=0.38, p=0.03\right)$, and nonflooded Arctic tundra lakes $\left(\log _{10} \mathrm{Chl}-a=0.07+0.50\right.$ $\log _{10} \mathrm{TP} ; R^{2}=0.39, p=0.07$; Fig. 7a). There was a strong relationship between TN and Chl- $a$ in forest-tundra lakes $\left(\log _{10} \mathrm{Chl}-a=-4.29+1.73 \log _{10} \mathrm{TN} ; R^{2}=\right.$ $0.64, p=0.01)$ and non-flooded Arctic-tundra lakes $\left(\log _{10} \mathrm{Chl}-a=-3.52+1.58 \log _{10} \mathrm{TN} ; R^{2}=0.68, p=\right.$ $0.01)$, but no relationship $\left(\log _{10} \mathrm{Chl}-a=0.65-0.06\right.$ $\log _{10} \mathrm{TN} ; R^{2}=0.006, p=0.80$; Fig. $7 \mathrm{~b}$ ) between TN and Chl- $a$ was observed in the flooded Arctic-tundra lakes.

\subsubsection{Multi-variable-Chl- $a$ relationships}

A GLM was used to assess the influence of multiple factors on Chl- $a$ production for all lakes and the three groups based on ecological zone and proximity to the Mackenzie River delta. The best model for all lakes revealed significant interactions between ecological zone and other independent variables, such as TN and TP (both $p \leq 0.018$ ). Consequently, the analyses for the factors affecting Chl- $a$ were performed for each of the three ecological lake types. Chl- $a$ in forest-tundra lakes was positively related to $\mathrm{TP}$ and negatively related to lake depth $\left(\log _{10} \mathrm{Chl}-a=0.21+0.79 \log _{10} \mathrm{TP}-0.34\right.$ $\log _{10}$ Depth; $\left.R^{2}=0.89, F=32.34, p=0.001\right)$. In nonflooded Arctic-tundra lakes, Chl- $a$ was associated positively with $\mathrm{TN}$ and negatively with conductivity $\left(\log _{10} \mathrm{Chl}-a=-2.49+1.56 \log _{10} \mathrm{TN}-0.49 \log _{10}\right.$ Cond; $\left.R^{2}=0.78, F=15.13, p=0.005\right)$, whereas Chl- $a$ was positively related to TP and negatively related to DOC and lake depth for flooded Arctic-tundra lakes $\left(\log _{10} \mathrm{Chl}-a=0.89+0.28 \log _{10} \mathrm{TP}-0.54 \log _{10} \mathrm{DOC}-\right.$ $0.33 \log _{10}$ Depth; $\left.R^{2}=0.88, F=27.68, p<0.0001\right)$. These results suggest that analyses assessing the factors affecting Chl- $a$ concentration should include variables beyond nutrients alone and that ecological zone and other supporting variables such as lake depth, DOC and conductivity be included in future analyses of Chl- $a$ in Arctic settings.

\section{DISCUSSION}

\subsection{Physical and chemical limnology}

Based on their physical characteristics and water chemistry, the lakes examined in this study resemble many other Canadian Arctic treeline lakes (Pienitz et al. 1997a, b; Rühland et al. 2003) as well as those in the Mackenzie River delta (Fee et al. 1988; Ramlal et al. 1991). Surface lakewater temperatures observed in our lake transect decreased from south to north with the warmest temperature $\left(17.3^{\circ} \mathrm{C}\right)$ recorded in the foresttundra lakes and the coldest $\left(12.9^{\circ} \mathrm{C}\right)$ in the flooded Arctic-tundra lakes. Pienitz et al. (1997a, b) also found 
that average surface water temperatures in the forest lakes was $4{ }^{\circ} \mathrm{C}$ warmer than those observed in tundra lakes. DO concentrations recorded during this study are relatively high and comparable to those reported in other shallow Arctic-tundra lakes (see Fee et al. 1988). Strong summer winds often result in complete lakewater mixing and oxygen-saturation of these shallow lakes (Fee et al. 1988), exceptions being Deep and Yaya lakes, which had well developed hypolimnions.

Measured major ions $\left(\mathrm{Na}, \mathrm{Cl}, \mathrm{Ca}\right.$, and $\left.\mathrm{SO}_{4}\right)$, turbidity and conductivity levels in this study showed identical trends of decreasing concentrations from the foresttundra to the Arctic-tundra lakes, with the highest values observed in the Mackenzie River delta floodplain lakes. In the present study, however, we believe that this variation is most likely due to differences in hydrology between floodplain and upland sites. More specifically, water from the Mackenzie River, which inundates the flooded Arctic-tundra lakes, is high in major ions, turbidity and conductivity. As well, increased major ion concentrations may be due to increased weathering activities and supply from the watershed (cf. Duff et al. 1999; Hay et al. 2000), and/or due to evaporative concentration of solutes (Keatley et al. 2007). Other studies conducted in the Mackenzie River delta and Tuktoyaktuk Peninsula lakes also reported relatively high conductivity, silica and major ions concentrations (Fee et al. 1988; Ramlal et al. 1991); much higher conductivity levels $\left(>c a 5000 \mu \mathrm{S} \mathrm{cm} ~^{-1}\right.$ ) have been found in the Husky Lakes due to marine influence (Evans \& Grainger 1980). Contrary to the above named studies, Pienitz et al. (1997a, b) found higher levels of major ions and DIC in boreal forest lakes compared to Arctictundra sites. Pienitz et al. (1997a, b) attributed this spatial pattern in their treeline studies in both Yukon and NT lakes to increased weathering and ion input from adjacent boreal forest watersheds due to higher precipitation and runoff.

Typical of subarctic and Arctic lakes, the majority of lakes sampled for this study were nutrient poor (TP often $\leq 10 \mu \mathrm{g} \mathrm{L}^{-1}$ ). In fact, approximately $30 \%$ of study lakes were ultraoligotrophic $\left(\leq 5 \mu \mathrm{g} \mathrm{L}^{-1}\right)$. In addition, the concentrations of dissolved inorganic nutrients such as $\mathrm{NO}_{3}+\mathrm{NO}_{2}, \mathrm{NH}_{3}, \mathrm{DP}, \mathrm{SiO}_{2}$ and ortho-P were exceedingly low or below detection limits at most lakes. Similar low levels of inorganic nutrients have been reported in several studies carried out in this region including lakes on the Tuktoyaktuk Peninsula (Anema et al. 1990; Ramlal et al. 1991), lakes in the Mackenzie River delta (Squires \& Lesack 2002), lakes located between southern Yukon and the Tuktoyaktuk Peninsula (Pienitz et al. 1997a), lakes situated between Yellowknife and Contwoyto Lake, NT (Pienitz et al. 1997b), Alaskan Toolik lakes (Levine \& Whalen 2001 and references therein) and Arctic Russia lakes (Duff et al. 1999). In a study conducted in lakes located in northern Russia on the Taimyr Peninsula, near the Lena River and Pechora
River, median concentrations of TP, $\mathrm{NH}_{3}$ and $\mathrm{SiO}_{2}$ were $5.4 \mu \mathrm{g} \mathrm{L}^{-1}, 5 \mu \mathrm{g} \mathrm{L}^{-1}$ and $1.30 \mathrm{mg} \mathrm{L}^{-1}$, respectively. While the above nutrient data would suggest co-N and P-limitation, TN:TP ratios (Fig. 3) derived from this study largely suggest P-limitation. High TN:TP ratios tend to prevail in oligotrophic lakes, because of dependence on catchment inputs which contain more $\mathrm{N}$ than $\mathrm{P}$ (Ogbebo et al. 2009 and references therein). Furthermore, according to the Redfield criteria (Redfield 1958), the PC:PP and PN:PP ratios showed P-limitation for both forest-tundra and non-flooded Arctic-tundra lakes. Nlimitation was inferred from the PC:PN ratios for only flooded Arctic-tundra lakes. The slight discrepancies between TN:TP and Redfield ratios could be a result of detrital interference on particulate ratios (Waiser \& Robarts 1995), especially in flooded lakes. Flanagan et al. (2003) found in their empirical analyses of published data that most Arctic lakes that were co-N and P-limited, generally have a TN:TP ratio $>14$. Concurrent N and P-limitation of algal standing stocks find support from studies conducted in other parts of this region (see Levine \& Whalen 2001 and references therein; Anema et al. 1990; Ramlal et al. 1991). Strong evidence for co$\mathrm{N}$ and P-limitation was found, irrespective of watershed vegetation types (tundra or forest) or elevation (flooded versus non-flooded), from our enrichment bioassay experiments conducted in a sub-set of lakes sampled for the present investigation (Ogbebo et al. 2009).

\subsection{Organic carbon and possible effects on resident biota}

DOC data from high latitude lakes are of immense ecological interest to aquatic ecologists (Duff et al. 1999). DOC can influence water chemistry and biota by binding to nutrients, acting as sunscreen against biologically harmful solar ultraviolet (UV-B) radiation, and even providing additional $\mathrm{C}$ for microbial plankton communities (Williamson et al. 1999). Consistent with other studies (Fee et al. 1988; Anema et al. 1990), the DOC levels observed in this study are moderately high (range 3.6-20.6 $\mathrm{mg} \mathrm{L}^{-1}$; mean $9.9 \mathrm{mg} \mathrm{L}^{-1}$ ) with relatively higher values in forest-tundra and flooded Arctic-tundra lakes than in non-flooded Arctic-tundra lakes. The above distribution in DOC levels reflects strong latitudinal changes in biome characteristics (i.e., trees versus tundra, degree of permafrost development, Mackenzie River flooding; Ramlal et al. 1991; Rühland et al. 2003; Keatley et al. 2007).

UV-B penetration has been reported to be entirely restricted to the surface waters by moderate levels of DOC $\left(\geq 4 \mathrm{mg} \mathrm{L}^{-1}\right)$, but increases exponentially with decreases in DOC (Schindler et al. 1990). Schindler et al. (1990) observed a decline in DOC concentrations during periods of increased warming and reduced annual precipitation in boreal lakes. Other studies have also shown that accelerated warming can change the quality and reduce the quantity of DOC through photo- 
bleaching (Ogbebo \& Ochs 2008). Consequently, a slight decline in DOC in our study lakes as a result of warming can cause UV-B, even at current ambient levels, to have adverse effects on the biota of our study lakes because these lakes are predominantly shallow, clear-waters with no deep-water refuge. As well, windinduced intermittent mixing in shallow lakes may cause plankton to be frequently exposed to harmful UV-B damage through regular circulation between deep and shallow layers of the epilimnion (Duff et al. 1999; Squires \& Lesack 2002; Ogbebo \& Ochs 2008). It is also plausible that increased light penetration may result in the proliferation of macrophytes and benthic algae (Schindler \& Smol 2006). High benthic algal production has been documented in many high latitude lakes (Sierszen et al. 2003; Bonilla et al. 2005).

POC:Chl- $a$ ratios were calculated in order to determine whether the $\mathrm{C}$ in sampled lakes was derived mostly from within-lake processes or terrestrial sources. An examination of the POC:Chl- $a$ ratio of our study sites showed that while some of the lakes derived their C from autochthonous sources or from both autochthonous and allochthonous sources (Fig. 5), a slight majority of the lakes are likely receiving most of their $\mathrm{C}$ from allochthonous sources. This result is consistent with the prevailing paradigm (Hamilton et al. 2001) and may be attributed to the fact that small lakes may experience considerable watershed impacts compared to large lakes. Hamilton and colleagues demonstrated that lakes in the Canadian Arctic Archipelago were largely dependent on non-phytoplankton $\mathrm{C}$ sources based on their POC:Chl- $a$ median value of 827.1. A comparably high POC:Chl- $a$ value was estimated from the data presented in Pienitz et al. (1997a, b); $\geq 600$ in lakes situated between Yellowknife and Contwoyto Lake and $\geq 340$ in sites that transect southern Yukon and Tuktoyaktuk Peninsula. In a related study, Rühland et al. (2003) found POC:Chl- $a$ ratios ranging from 170:1-2973:1, with a mean of 616:1 in 33 sites located along a transect from Yellowknife to Bathurst Inlet. A small fraction of the POC may however be generated internally from other sources such as macrophytes, epiphytes, and benthic algae (Squires \& Lesack 2003). Caution should however be taken in interpreting POC:Chl- $a$ data as organic detritus may sometimes affect the POC values (Waiser \& Robarts 1995). Furthermore, organic detritus may persist for a longer period of time in these colder subarctic lakes than in temperate systems.

\subsection{Principal components analysis}

The distribution of variables and sites in the ordination closely track patterns across the three ecological zones and proximity to the Mackenzie River (i.e., elevation) discussed earlier. The first axis of the PCA effectively contrasted the forest-tundra and Arctic-tundra lakes. The Arctic-tundra lakes were further separated into two groups, the non-flooded and the flooded lakes, reflecting variability in water chemistry and physical conditions within this zone. Most of the more productive, small, shallow and turbid lakes plotted almost exclusively on the upper half of PC2. This outcome suggests that there were several gradients of variation in the physical and chemical data of these sites along the latitudinal gradient and agrees with the earlier findings of Pienitz et al. (1997a). Several other studies including Pienitz et al. (1997a) and Rühland et al. (2003) have also used PCA to clearly separate forested sites from the lakes located in treeless watersheds (i.e., Arctic-tundra and alpine-tundra sites). Our study, like that of Rühland et al. (2003), showed that freshwater ecosystems situated on opposite sides of the Canadian Arctic treeline exhibit relatively distinct differences in limnological characteristics. Rühland et al. (2003) observed that the concentrations of most variables such as major ions, nutrients, DIC, DOC, temperature and conductivity were highest in forested catchments and tended to decrease with increasing latitude, results relatively similar to this study.

\subsection{Models of Chl-a}

The anticipated increases in temperature (global warming) and nutrient loading (watershed disturbance; Rouse et al. 1997; Lim et al. 2001; Smith et al. 2005), are expected to produce a dramatic increase in algal productivity and standing stocks (Flanagan et al. 2003; Keatley et al. 2007). Eutrophication of aquatic ecosystems due to nutrient loading can have an adverse effect on water quality, including undesirable phytoplankton blooms, reduced water transparency, reduced oxygen content and fish kills from anoxia (Edmondson 1991; Schindler et al. 2008). Future management of these ecosystems therefore requires models that allow accurate prediction of the effects of both nutrients and increased temperature or their interactions on phytoplankton (Attayde \& Bozelli 1999; Flanagan et al. 2003). The analysis of empirical relationships of ecological variables across ecosystems is a valuable approach for the generation of hypotheses regarding cause-effect mechanisms, as well as for ecosystem management.

While there was a large variability in the correlation between Chl- $a$, TP, and TN in the present study, TP explained relatively more of the variance in Chl- $a$ than TN. Most of the regression coefficients were significant $(p<0.05)$ except TN-Chl- $a$ for flooded Arctic-tundra lakes. Overall, our results are consistent with the traditional TP-Chl- $a$ paradigm (see Dillon \& Rigler 1974; Schindler 1977; Smith 1982), but the magnitude of these regressions vary greatly from published studies on a variety of temperate lakes (e.g., McCauley 1989). In those studies, the r-square value was $\geq 0.61$ for both TPChl- $a$ and TN-Chl- $a$. The lack of a linear relationship between Chl- $a$ and TN or weak correlation between Chl- $a$ and TP in flooded lakes, though not unexpected, would suggest that other factors beyond nutrients, such 
as light, may be limiting algal biomass in these systems. Since many of the floodplain lakes were small and shallow with very low residence time, the effects of the sediment laden Mackenzie River waters on primary productivity could be considerable (Spears \& Lesack 2006).

High latitude aquatic biota may respond slightly differently to nutrient addition than temperate systems because of their exposure to low energy, low temperature, and extreme variations in daylength (Flanagan et al. 2003). These abiotic factors often constrain plankton nutrient uptake, utilization, growth and biomass accumulation (Duff et al. 1999; Keatley et al. 2007). In the present study, the slope of the TP-Chl- $a$ relationship for the forest-tundra lakes compared to the Arctic-tundra lakes was different from TN-Chl- $a$. We hypothesize that a combination of factors, including temperature, phytoplankton composition, zooplankton grazing and heterotrophic grazing, or their interactions, may account for this variation. It is therefore not surprising that a weak correlation between TP and Chl- $a$, which may suggest the limitation of primary productivity by non-nutrient related factors, has been documented in field surveys (Pienitz et al. 1997b; Duff et al. 1999; Rühland et al. 2003) and empirical analyses of published data (Flanagan et al. 2003).

Because DOC acts as the major driver of material and energy fluxes in aquatic ecosystems (Williamson et al. 1999), we hypothesized that factors such as water temperature, nutrients (TP, TN), and DOC may combine to regulate algal standing stocks in subarctic and Arctic lakes. Consequently, we used GLM to examine these and other factors that may control algal biomass. There was however great variability in models derived for the three groups of lakes. The GLM for forest-tundra lakes showed that Chl- $a$ is positively related to TP and inversely related to depth, while in the flooded Arctictundra lakes Chl- $a$ was positively related to TP, but negatively related to depth and DOC. In non-flooded Arctic-tundra lakes, Chl- $a$ was positively related to TN and negatively related with conductivity. Taken together, our ability to predict algal biomass was greatly enhanced when other variables that affect water clarity such as DOC, depth and conductivity (depending on data cluster) were incorporated into a multivariate nutrient-based model. This outcome is not unexpected because nutrient effects on phytoplankton are influenced by lake morphology (mean depth, area, and hydrology) and drainage watershed (Rühland et al. 2003). For instance, in most shallow lakes (i.e., the majority of our study lakes), the effects of terrestrial inputs are more profound on the ecological processes compared to larger lakes because of the large drainage basin to lake surface area ratio(cf. Spears \& Lesack 2006). Moreover, moderate to high levels of DOC have been found to significantly modulate nutrient cycling either indirectly through changes in light attenuation and thermal strati- fication, or directly by simply binding to nutrients and making them unavailable (see Williamson et al. 1999 for list of processes influenced by DOC). If the results of this study are representative of many other high latitude freshwater systems, then the control of eutrophication which traditionally requires reduced $\mathrm{P}$ influx into lakes (Edmonson 1991; Schindler et al. 2008), should also give prominent consideration to $\mathrm{N}$ as well as other factors such as DOC and lake depth (Rühland et al. (2003).

\section{CONCLUSIONS}

This study provides a description of the physical and chemical characteristics of lakes situated in the Mackenzie River delta and along the eastern side of the Mackenzie River. Several trends in water chemistry were observed. Conductivity, turbidity and major ions $\left(\mathrm{Na}, \mathrm{Cl}, \mathrm{Ca}\right.$, and $\left.\mathrm{SO}_{4}\right)$ concentrations were highest in the floodplain lakes and strongly related to elevation and proximity to the Mackenzie River. The floodplain lakes chemical data were highly influenced by flood water from the Mackenzie River (Hay et al. 2000; Spears \& Lesack 2006). The majority of lakes sampled for this study were nutrient poor (TP often $<10 \mu \mathrm{g} \mathrm{L}^{-1}$ ). Concentrations of dissolved inorganic nutrients such as $\mathrm{NO}_{3}+\mathrm{NO}_{2}, \mathrm{NH}_{3}$, DP, and ortho-P were exceedingly low or undetectable in the surface waters at most sites. In this study we found three different predictors of Chl- $a$ in the three groups of lakes. The commonly used P-Chl$a$ relationship for most temperate systems was inadequate for high latitude lakes. The capacity to predict algal biomass was enhanced when other variables that affect water quality, such as DOC, depth and conductivity, were incorporated into a multivariate model. To further shed light on the variation in Chl- $a$, we are currently analyzing data on phytoplankton composition and zooplankton abundance from study lakes.

Global warming is likely to have both direct and indirect effects on phytoplankton. For instance, increased nutrient or organic matter inputs as a result of watershed disturbance (e.g., with resource exploitation and/or permafrost thawing) in combination with warmer temperatures and longer growing season may dramatically enhance algal standing stocks and primary productivity (Smith et al. 2005; Smol et al. 2005). As well, increased nutrient and organic matter export into lakes may deplete oxygen concentrations in deep stratified lakes such as Deep and Yaya and/or all lakes during winter (Ogbebo et al. 2009). Consequently, close attention must be paid to potential interaction of ongoing development and global warming. With the possible development of the MGP pipeline, we believe that more food web and long-term monitoring studies that involve exploring the mechanisms controlling nutrient uptake and recycling in the vast treeline region of northern Canada are needed. These data will provide detailed information on the natural variability of these lakes and 
allow for accurate prediction of the effects of development and climate warming on aquatic ecosystems (Pienitz et al. 1997a, b).

\section{ACKNOWLEDGEMENTS}

This study was supported by Environment Canada through MGP funding to M. S. Evans. The authors thank Xiaowa Wang for her laboratory assistance and Megan Kroes and Chantel Showers for their help in the field. We are grateful to the Gwich'in Renewable Resource Board and Inuvialuit Renewable Resource Committees for their support, and the Aurora Research Institute for laboratory support. Thanks also are extended to the analytical laboratories involved in this study including NLET-Saskatoon, NLET-Burlington, and PNLET-Edmonton. Special thanks go to Voyage Air for excellent float plane support during our field sampling.

\section{REFERENCES}

ACIA. 2005. Arctic Climate Impact Assessment. Cambridge University Press, NY. $1024 \mathrm{pp}$

Anema, C., R.E. Hecky, E.J. Fee, D. Nernberg \& S.J. Guildford.1990. Water chemistry of some lakes and channels in the Mackenzie Delta and on the Tuktoyaktuk Peninsula, N. W. T., 1985. Can. Data Rep. Fish. Aquat. Sci., 726.

Attayde, J.L. \& R.L. Bozelli, 1999. Environmental heterogeneity patterns and predictive models of chlorophyll- $a$ in a Brazillian coastal lagoon. Hydrobiologia, 390: 129-139.

Bonilla, S., V. Villeneuve \& W.F. Vincent. 2005. Benthic and planktonic algal communities in a high arctic lake: pigment structure and contrasting response to nutrient enrichment. J. Phycol., 41: 1120-1130.

Dillon, P. J. \& F. H. Rigler. 1974. The phosphorus-chlorophyll relationship in lakes. Limnol. Oceanogr., 19: 767-773.

Duff, K.E., T.E. Laing, J.P. Smol \& D.R.S. Lean. 1999. Limnological characteristics of lakes located across arctic treeline in northern Russia. Hydrobiologia, 391: 205-222.

Edmondson, W.T. 1991. The use of ecology: Lake Washington and beyond. Univ. Washington. 329 pp. ISBN 0-25997024-3.

Elser, J.J., M.E.S. Bracken, E.E. Cleland, D.S. Gruner, W.S. Harpole, H. Hillebrand, J.T. Ngai, E.W. Seabloom, J.B. Shurin \& J.E. Smith. 2007. Global analysis of nitrogen and phosphorus limitation of primary producers in freshwater, marine and terrestrial ecosystems. Ecology Letters, 10: $1135-1142$.

Environment Canada. 1992. Analytical methods manual. Inland Waters Directorate, Water Quality Branch, Environ. Canada.

Environment Canada. 2005. Canadian Climate Normals or Averages 1971-2000, http://www.climate.weatheroffice. ec.gc.ca/climate_normals/index_e.html [accessed November 26 2008]

Eppley, R.W., W.G. Harrison, S.W. Chisholm \& E. Stewart. 1977. Particulate organic matter in surface waters off Southern California and its relationship to phytoplankton. J. Mar. Res., 35: 671-696.

Evans, M.S., M.T. Arts \& R.D. Robarts. 1996. Algal productivity, algal biomass, and zooplankton biomass in a phosphorus-rich, saline lake: deviations from regression model predictions. Can. J. Fish. Aquat. Sci., 53: 1048-1060.

Evans, M.S. \& E.H. Grainger. 1980. Zooplankton in a Canadian Arctic Estuary. In: Estuarine Perspectives. ISBN 012-404060-8: 199-210.
Evans, M.S., F. Ogbebo, M. Waiser, D. Barton, M. Davies, B. Kilgeour, D. Halliwell \& J. Keating. 2007. Biological studies as part of investigations with the Mackenzie Gas Pipeline Environmental Assessment with comparisons to ongoing studies with the Regional Aquatics Monitoring Program. 60th Annual Canadian Water Resources Association Conference. Saskatoon, SK. June 25-28, 2007: 10-11.

Fee, E.J., R.E. Hecky, S.J. Guildford, C. Anema, D. Mathew \& K. Hallard. 1988. Phytoplankton primary production and related limnological data for lakes and channels in the Mackenzie Delta and lakes on the Tuktoyaktuk Peninsula, N.W.T. Canadian Technical report of Fisheries and Aquatic Science, No. 1614.

Flanagan, K., E. McCauley, T. Prowse \& F. Wrona. 2003. Climate Change: The potential for latitudinal effects on algal biomass in aquatic systems. Can. J. Fish. Aquat. Sci., 60: 635-639.

Hamilton, P.B., K. Gajewski, D.E. Atkinson \& D.R.S Lean. 2001. Physical and chemical limnology of 204 lakes from the Canadian Arctic Archipelago. Hydrobiologia, 457: $133-148$

Hay, M.B., N. Michelutti \& J.P. Smol. 2000. Ecological patterns of diatom assemblages from Mackenzie Delta lakes, Northwest Territories, Canada. Can. J. Bot., 78: 19-33.

Imperial Oil Resources Ventures Limited. 2004. Environmental Impact Statement for the Mackenzie Gas Project. Volume 3: Biophysical Baseline. Part B Aquatic Resources: Groundwater, Hydrology and Water Quality. Submitted to: National Energy Board and to the Joint Review Panel [online]. Available from http://www. ngps.nt.ca/applicationsubmission/EIS.html [accessed 05 January 2009].

Jackson, D.A. 1993. Stopping rules in principal components analysis: A comparison of heuristical and statistical approaches. Ecology, 74: 2204-2214.

Keatley, B.E., M.S.V. Douglas \& J.P. Smol. 2007. Physical and chemical characteristics of lakes and ponds across environmental gradients on Melville Island, Nunavut, N.W.T., High Arctic Canada. Fundamental and Applied Limnology, 168: 355-376.

Levine, M.A. \& S.C. Whalen. 2001. Nutrient limitation of phytoplankton production in Alaskan Arctic foothill lakes. Hydrobiologia, 455: 189-201.

Lim, D.S.S., M.S.V. Douglas, J.P. Smol \& D.R.S. Lean. 2001. Physical and chemical limnological characteristics of 38 lakes and ponds on Bathurst Island, Nunavut, Canadian High Arctic. Int. Rev. Hydrobiol., 86: 1-22.

Mackenzie River Basin Board (MRBB). 2004. Mackenzie River Basin: State of the Aquatic Ecosystem Report 2003. Mackenzie River Basin Board, Fort Smith, Northwest Territories, Canada.

Manly, B.F.J. 1986. Multivariate statistical methods: A primer. Chapman and Hall, New York.

McCauley, E., J.A. Downing \& S. Watson. 1989. Sigmoid relationships between nutrients and chlorophyll among lakes. Can. J. Fish. Aquat. Sci., 46: 1171-1175.

Nusch, E.A. 1980. A comparison of different methods for chlorophyll and pheopigment analysis. Ergeb. Limnol., 14: 14-36.

Ogbebo, F.E., M.S. Evans, M. Waiser, T. Vijay \& J. Keating. 2009. Nutrient limitation of phytoplankton growth in Arctic lakes of the lower Mackenzie River Basin, Northern Canada. Can. J. Fish. Aquat. Sci., 66: 247-260; doi:10.1139/F08-202.

Ogbebo, F.E. \& C.A. Ochs. 2008. Bacterioplankton and Phytoplankton Production Rates Compared at Different Levels of Solar Ultraviolet Radiation and Limiting Nutrient Ratios. J. Plankton Res., 30(11): 1271-1284; doi: 10.1093/plankt/fbn083.

Pienitz, R., J.P. Smol \& D.R.S. Lean. 1997a. Physical and chemical limnology of 59 lakes located between the 
southern Yukon and the Tuktoyaktuk Peninsula, Northwest Territories (Canada). Can. J. Fish. Aquat. Sci., 54: 330-346.

Pienitz, R., J.P. Smol \& D.R.S. Lean. 1997b. Physical and chemical limnology of 24 lakes located between Yellowknife and Contwoyto Lake, Northwest Territories (Canada). Can. J. Fish. Aquat. Sci., 54: 347-358.

Prowse, T.D., F.J. Wrona, J.D. Reist, J.E. Hobbie, L.M.J. Lévesque \& W.F. Vincent. 2006. General features of the Arctic relevant to climate change in freshwater ecosystems. Ambio, 35: 330-338.

Ramlal, P.S., C. Anema, E. Cummings, E.J. Fee, S.J. Guildford, K. Hallard, R.E. Hecky, R.H. Hesslein, S. Himmer, H. Kling, D. Matthew, D. Nernberg \& E. Schindler. 1991. Macrophytes and Algal Studies of Some Lakes in the Mackenzie Delta and on the Tuktoyaktuk Peninsula, 19851986. Can. Data Rep. Fish. Aquat. Sci., 839.

Redfield, A.C. 1958. The biological control of chemical factors in the environment. Am. Sci., 46: 205-222.

Rouse, W.R., M.S.V. Douglas, R.E. Hecky, A.E. Hershey, G.W. Kling, L. Lesack, P. Marsh, M. McDonald, B.J. Nicholson, N.T. Roulet \& J.P. Smol. 1997. Effects of climate change on the freshwaters of Arctic and Subarctic North America. Hydrological Processes, 11: 873-902.

Rühland, K.M., J.P. Smol, X. Wang \& D.C.G. Muir. 2003. Limnological characteristics of 56 lakes in the Central Canadian Arctic Treeline Region. J. Limnol., 62(1): 9-27.

Sakamoto, M. 1966. Primary production by the phytoplankton community in some Japanese lakes and its dependence on lake depth. Arch. Hydrobiol., 62: 1-28.

Sankoh, A.J., M.F. Huque \& S.D. Dubey. 1997. Some comments on frequently used multiple endpoint adjustment methods in clinical trials. Statistics in Medicine, 16: 25292542.

Schindler, D.W. 1977. Evolution of phosphorus limitation in lakes. Science, 195: 260-262.

Schindler, D.W., K.G. Beaty, E.J. Fee, D.R. Cruikshank, E.R. Debruyn, D.L. Findlay, G.A. Linsey, J.A. Shearer, M.P. Stainton \& M.A. Turner. 1990. Effects of climatic warming on lakes of the central boreal forest. Science, 250: 967970 .

Schindler, D.W. \& J.P. Smol. 2006. Cumulative effects of climate warming and other human activities on freshwaters of Arctic and Subarctic North America. Ambio, 35: 160-168.

Schindler, D.W., R.E. Hecky, D.L. Findlay, M.P. Stainton, B.R. Parker, M.J. Paterson, K.G. Beaty, M. Lyng. \& S.E.M. Kasian. 2008. Eutrophication of lakes cannot be controlled by reducing Nitrogen input: Results of a 37year whole-ecosystem experiment. Proceedings of the National Association of Sciences (PNAS), 105(32): 1125411258.

Sierszen, M.E., M.E. McDonald \& D.A. Jensen. 2003. Benthos as the basis for arctic lake food webs. Aquatic Ecology, 37: 437-445.

Smith, L.C., Y. Sheng, G.M. MacDonald \& L.D. Hinzman. 2005. Disappearing arctic lakes. Science, 308 (5727), 1429.

Smith, V.H. 1982. The nitrogen and phosphorus dependence of algae in lakes: an empirical and theoretical analysis. Limnol. Oceanogr., 27: 1101-1112.

Smol, J.P., A.P. Wolfe, H.J.B. Birks, M.S.V. Douglas, V.J. Jones, A. Korholai, R. Pienitz, K. Rühland, S. Sorvari, D. Antoniades, S.J. Brooks, M. Fallu, M. Hughes, B.E. Keatley, T.E. Laing, N. Michelutti, L. Nazarova, M. Nyman, A. M. Paterson, B. Perren, R. Quinlan, M. Rautio, É. Saulnier-Talbot, S. Siitonen, N. Solovieva \& J. Weckström. 2005. Climate-driven regime shifts in the biological communities of Arctic lakes. PNAS, 102: 43974402 .

Sokal, R.R. \& F.J. Rohlf. 1995. Biometry: The principles and practice of statistics in biological research. $3^{\text {rd }}$ Ed. W.H. Freeman and Co., New York.

Spears, B.M. \& L.F.W. Lesack. 2006. Bacterioplankton production, abundance, and nutrient limitation among lakes of the Mackenzie Delta (Western Canadian Arctic). Can. J. Fish. Aquat. Sci., 63: 845-857.

Squires M.M. \& L.F.W. Lesack. 2002. Water transparency and nutrients as controls on phytoplankton along a floodfrequency gradient among lakes of the Mackenzie Delta, western Canadian Arctic. Can. J. Fish. Aquat. Sci., 59: 1339-1349.

Squires M.M. \& L.F.W. Lesack. 2003. The relation between sediment nutrient content and macrophyte biomass and community structure along a water transparency gradient among lakes of the Mackenzie Delta. Can. J. Fish. Aquat. Sci., 60: 333-343.

ter Braak, C.J.F. \& I.C. Prentice. 1988. A theory of gradient analysis. Adv. Ecol. Res., 18: 271-317.

Waiser, M.J. \& R.D. Robarts. 1995. Microbial nutrient limitation in prairie saline lakes with high sulfate concentration. Limnol. Oceanogr., 40: 566-574.

Williamson, C., D. Morris \& O. Olson. 1999. Dissolved organic carbon and nutrients as regulators of lake ecosystems: resurrection of a more integrated paradigm. Limnol. Oceanogr., 44: 795-803. 